\section{Performance Analysis of Wavefront Curvature Methods for Range Estimation of a Moving Source}

JEAN-PIERRE LE CADRE, Member, IEEE IRISA/CNRS

France

In numerous practical situations a large array of sensors is required, especially for improving the system ability to detect and track moving sources. However, the array performance is strongly affected by the lack of stationarity of the impinging signals. The use of a large array of sensors allow us to perform the source localization by using the wavefront curvature. The analysis of the range estimates based on the wavefront curvature have been thoroughly performed by various authors but, to our knowledge, is restricted to the case of fixed sources.

The study of the effects of source motion for range estimation (Section III) and source detection (Section V) constitutes an important part of this work. This study reveals that the array performance can be far from the expected values, especially for large array. Therefore, the robustness of a direct processing of the whole sensor array is very questionable. The computational burden constitutes another serious drawback of this (direct) approach.

The use of partitioned processings (Sections IV and VI) seems thus quite promising even if (and perhaps because) it is not optimal so as to fulfil the computation cost and robustness requirements. The performance of such processings for estimating the range of moving sources is presented.

Manuscript received November 29, 1993; revised June 14, 1994. IEEE Log No. T-AES/31/3/09757.

Author's address: IRISA/CNRS, Campus de Beaulieu, 35042 Rennes Cedex, France.

$\overline{0018-9251 / 95 / \$ 4.00 ~(C) ~} 1995$ IEEE

\section{INTRODUCTION}

In numerous practical situations a large array of sensors is required, especially for improving the system ability to detect and track moving sources. However, the array performances are strongly affected by the lack of stationarity of the impinging signals which is due to multiple factors like time-varying source models, medium, and array. Here, we are chiefly concerned with the effects of the source motions on the array processing performance.

The use of a large array of sensors allow us to perform the source localization by using the wavefront curvature. It is, typically, an instantaneous localization method since it assumes that the received signals are stationary during the observation time. On the opposite, the bearings-only target motion analysis methods rely upon a nonstationary source model. As it is shown, the performance of wavefront curvature methods may be greatly affected by the source motions.

The study of the effects of source motions for range estimation (Section III) and source detection (Section V) constitutes a large part of this work. An analytical expression of the variance of the estimated range of a moving source is obtained and, then, validated by comparing it with simulation results. The integration time (or equivalently the number of snapshots) appears to be instrumental for optimizing the variance of the estimated range. Obviously, it depends on the source kinematic parameters which are generally unknown but, overall, it is directly related to the array length.

These conclusions are also valid for optimizing the detection performance, studied in Section V. This study reveals that the array performance can be far to the expected values, especially for large array and, in particular, are strongly degraded by the source motions. This is especially true for multiple moving sources since it is not possible to optimize the integration time for all the sources simultaneously. Furthermore, the robustness of a direct processing of the whole sensor array is very questionable (bias in cross-spectral estimation, wavefront coherence). The computational burden constitutes another drawback of this (direct) approach.

The use of partitioned processings (Sections IV and VI) seems thus quite promising, even if-and perhaps because-it is not an optimal processing in order to fulfil the computation cost and robustness requirements. A natural approach for partitioned processing consists in considering (partitioned) pre-processing of a large array and the related source bearing estimates, these estimates being then associated in order to obtain a source range estimation. It constitutes a simplistic approach of the much more general problem: is it possible to exchange the angular performance (variance of the estimated bearings) against increased range performance? 


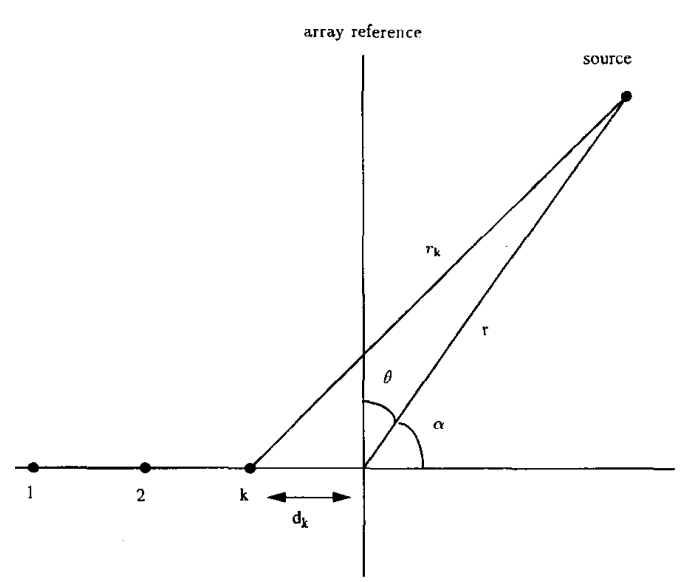

Fig. 1. Definition of geometric parameters.

The position of the general problem of source localization is outlined in Section II, as well as some fundamental quantities namely $2 \theta_{3}$ and $2 r_{3}$. Section III deals with the estimation of the range of a moving source by using the wavefront curvature and a direct approach. The same problem is considered in Section IV but, this time, with a partitioned array processing. Its performances are compared with those of the direct (and classical) approach.

Section $V$ is devoted to the study of the detection performance for moving sources. The problems related to bearings association are considered in Section VI.

\section{THE GENERAL PROBLEM OF SOURCE LOCALIZATION BY USING THE WAVEFRONT CURVATURE, DEFINITIONS AND NOTATIONS}

For the rest of this work, the array is assumed to be linear and constituted of $p$ equispaced sensors. The elementary intersensor distance is denoted by $d$, $d$ is chosen equal to $\lambda / 2$ ( $\lambda$ : wavelength). The source position is defined by using polar coordinates for instance with respect to (wrt) the array center, the corresponding angles and parameters are defined below (Fig. 1).

The bearing angles $\theta$ are referenced wrt the perpendicular of the array axis, and the source distance $r$ is defined as the distance of the source from the array center (see Fig. 1). The distance $r_{k}$ of a source, whose polar coordinates are $(\theta, r)$, from the $k$ th sensor is deduced from classical trigonometry, i.e.,

$$
\begin{aligned}
r_{k}^{2} & =d_{k}^{2}+r^{2}+2 d_{k} r \cos \alpha \\
& =d_{k}^{2}+r^{2}+2 d_{k} r \sin \theta
\end{aligned}
$$

so that

$$
r_{k}=\left(r^{2}+d_{k}^{2}+2 r d_{k} \sin \theta\right)^{1 / 2}
$$

with

$$
\begin{aligned}
& d_{k}=\frac{p-1}{2} \cdot d-(k-1) d=d \cdot\left(\frac{p-1}{2}-k+1\right) \\
& (p: \text { odd }) .
\end{aligned}
$$

Especially for the performance calculation, the narrowband frame allow us to use the linear algebra formalism, reducing thus the notational complexity. Since this formalism is now classical $[1,2]$ it is not detailed further.

Let $D_{\theta_{0}, r_{0}}$ be the steering vector [2] associated with a source $\left(\theta_{0}, r_{0}\right)$ and defined below:

$$
D_{\theta_{0}, r_{0}}=\left(1, \ldots, \exp \left(2 i \pi f \tau_{k}\left(\theta_{0}, r_{0}\right)\right), \ldots\right)^{\mathrm{t}}
$$

(the symbol $\mathrm{t}$ meaning transposition) with

$$
\mid \begin{aligned}
& \tau_{k}: \text { time delay from the } 1 \text { st sensor to the } k \text { th } \\
& i^{2}=-1 \\
& f: \text { frequency. }
\end{aligned}
$$

The time delay $\tau_{k}\left(\theta_{0}, r_{0}\right)$ corresponds to the propagation of the acoustical wave in a nondispersive medium and is directly deduced from (1), i.e.,

$$
\begin{aligned}
& \tau_{k}\left(\theta_{0}, r_{0}\right)=\frac{1}{c}\left[\left(r_{0}+d_{k}^{2}+2 r_{0} d_{k} \sin \theta_{0}\right)^{1 / 2}-r_{0}\right] \\
& \text { c: celerity. }
\end{aligned}
$$

The array directivity (power beam pattern) is the two-dimensional functional defined below:

$$
\begin{aligned}
f(\theta, r) & =\left|D_{\theta, r}^{*} D_{\theta_{0}, r_{0}}\right|^{2} \\
& =\left|\sum_{k=1}^{p} \exp \left[2 i \pi f\left(\tau_{k}\left(\theta_{0}, r_{0}\right)-\tau_{k}(\theta, r)\right)\right]\right|^{2}
\end{aligned}
$$

$\left({ }^{*}\right.$ : transposition and conjugation, $\mid$ |: complex modulus).

This functional $f(\theta, r)$ presents a global maximum for the exact values of the parameters $(\theta, r)$ (i.e., $\theta=$ $\theta_{0}, r=r_{0}$ ). The parameters $2 \theta_{3}$ and $2 r_{3}$ are defined as the half-beamwidth, respectively, in bearing and distance. Even if they correspond to a deterministic analysis, they condition greatly the array performance in angular and range resolution. Neglecting the wavefront curvature (plane wave hypothesis), the following expression of $2 \theta_{3}$ is obtained by using a second-order expansion of $f(\theta, r)$ and known as the Rayleigh formula [1, 2]:

$$
2 \theta_{3}=\frac{\sqrt{6}}{\pi}\left(\frac{p-1}{p+1}\right)^{1 / 2} \frac{\lambda}{L} \frac{1}{\cos \theta_{0}}
$$$$
L \text { : array length. }
$$

A second-order approximation of $2 \theta_{3}$ and $2 r_{3}$ is obtained by using the same way, more precisely:

$$
\begin{aligned}
f(\theta, r) \stackrel{2}{=} & f\left(\theta_{0}, r_{0}\right)+\left(\Omega-\Omega_{0}\right)^{\mathrm{t}} \nabla f\left(\Omega_{0}\right) \\
& +\frac{1}{2}\left(\Omega-\Omega_{0}\right)^{\mathrm{t}} H\left(\Omega_{0}\right)\left(\Omega-\Omega_{0}\right)
\end{aligned}
$$


with

$$
\begin{aligned}
& \stackrel{2}{=}: \text { second-order expansion } \\
& \nabla f: \text { gradient vector } \\
& H: \text { Hessian matrix. }
\end{aligned}
$$

The calculation of $2 \theta_{3}$ and $2 r_{3}$ requires the calculation of the Hessian matrix $H\left(\Omega_{0}\right)$. For that aim, the steering vector $D(\theta, r)$ (in (2)) is advantageously written in the following form:

$$
D_{\theta, r}=\left(\exp \left(i k h_{1}\right), \ldots, \exp \left(i k h_{p}\right)\right)^{\mathrm{t}}
$$

with

$$
\mid \begin{aligned}
i^{2} & =-1 \\
k & =2 \pi / \lambda=2 \pi f / c \\
h_{j} & =\left(r^{2}+d_{j}^{2}+2 d_{j} r \sin \theta\right)^{1 / 2}-r \\
d_{j} & =\frac{p-1}{2} d-(j-1) d \quad 1 \leq j \leq p .
\end{aligned}
$$

In (7), the scalar $k$ represents the wavenumber while the functions $h_{j}$ represent the difference of path length from the source to the various sensors.

With these notations, the functional $f(\theta, r)$ stands as follows:

$$
f(\theta, r)=\sum_{j=1}^{p} \exp \left[i k\left(h_{0 j}-h_{j}\right)\right]
$$

and, the Hessian matrix at the point $\left(\theta_{0}, r_{0}\right)$ is given by

$$
\begin{aligned}
\frac{\partial^{2} f}{\partial \theta^{2}} & =2 A A^{\prime \prime}+2\left(B^{\prime}\right)^{2} \\
\frac{\partial^{2} f}{\partial r^{2}} & =2 A^{o o}+2\left(B^{o}\right)^{2} \\
\frac{\partial^{2} f}{\partial \theta \partial r} & =2 A A^{o \prime}+2 B^{\prime} B^{o}
\end{aligned}
$$

with

$$
\mid \begin{aligned}
& A=p, \\
& A^{\prime \prime}=-k^{2} \sum_{i=1}^{p}\left(\frac{\partial h_{i}}{\partial \theta}\right)^{2} \\
& A^{o o}=-k^{2} \sum_{i=1}^{p}\left(\frac{\partial h_{i}}{\partial r}\right)^{2} \\
& B^{\prime}=-k \sum_{i=1}^{p}\left(\frac{\partial h_{i}}{\partial \theta}\right) \\
& B^{o}=-k \sum_{i=1}^{p} \frac{\partial h_{i}}{\partial r} \\
& A^{o \prime}=-k^{2} \sum_{i=1}^{p}\left(\frac{\partial h_{i}}{\partial \theta}\right)\left(\frac{\partial h_{i}}{\partial r}\right) \\
& B^{o \prime}=-k^{2} \sum_{i=1}^{p}\left(\frac{\partial^{2} h_{i}}{\partial \theta \partial r}\right) .
\end{aligned}
$$

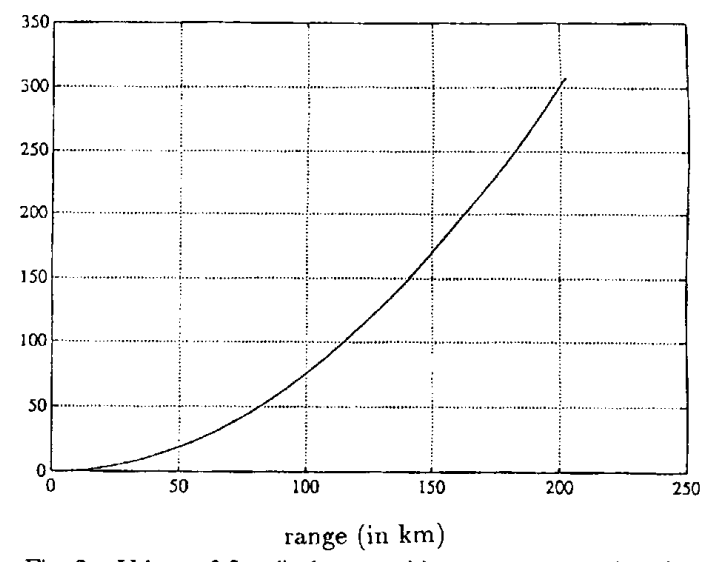

Fig. 2. Values of $2 r_{3}$ (in $\mathrm{km}, y$-axis) versus range $r$ ( $x$-axis, in $\mathrm{km}$ ) for source in array broadside $(\theta=\pi / 2)$.

Numerical considerations show that the terms $\partial h_{i} / \partial r$ are little in regard to the terms $\partial h_{i} / \partial \theta$, so that the matrix $H$ has roughly the following form:

with

$$
H=\left(\begin{array}{ll}
G & S \\
S & \varepsilon
\end{array}\right)
$$

$$
\varepsilon \ll S \quad \text { and } \quad S \ll G .
$$

Consider now the diagonalization of $H$, i.e.,

$$
H=P\left(\begin{array}{cc}
\lambda_{1} & 0 \\
0 & \lambda_{2}
\end{array}\right) P^{-1}
$$

( $\lambda_{1}$ and $\lambda_{2}$ eigenvalues, $P$ orthogonal) then, direct calculations provide the following approximations:

and

$$
\lambda_{1} \simeq G+\frac{S^{2}}{G-\varepsilon} \quad \lambda_{2} \simeq \varepsilon-\frac{S^{2}}{G-\varepsilon}
$$

$$
P \simeq\left(\begin{array}{cc}
1 & \frac{S}{G-\varepsilon} \\
\frac{S}{\varepsilon-G} & 1
\end{array}\right) .
$$

According to (9), the matrix $P$ is approximately diagonal and consequently $H$ can be approximated by $\Lambda$. Collecting (6), (8), and (9) the following approximations of $2 \theta_{3}$ and $2 r_{3}$ are thus obtained

$$
\begin{aligned}
& 2 \theta_{3} \simeq \frac{2 p}{\left(\frac{\partial^{2} f}{\partial \theta^{2}}\right)^{1 / 2}} \quad 2 r_{3} \simeq \frac{2 p}{\left(\frac{\partial^{2} f}{\partial r^{2}}\right)^{1 / 2} .} \\
& \frac{\partial^{2} f}{\partial \theta^{2}} \quad \text { and } \quad \frac{\partial^{2} f}{\partial r^{2}} \quad \text { given by (8). }
\end{aligned}
$$

The calculation of $2 \theta_{3}$ and $2 r_{3}$ is illustrated by Figs. 2 and 3. The value of $2 r_{3}$ is plotted on the $y$-axis, whereas the corresponding value of $r$ is plotted on the $x$-axis. Fig. 2 corresponds to a source situated at the bearing 0 (from the array broadside), and Fig. 3 to a bearing $\theta=\pi / 4$. As expected, the lowest values of $2 r_{3}$ are obtained for a source in the array broadside. 


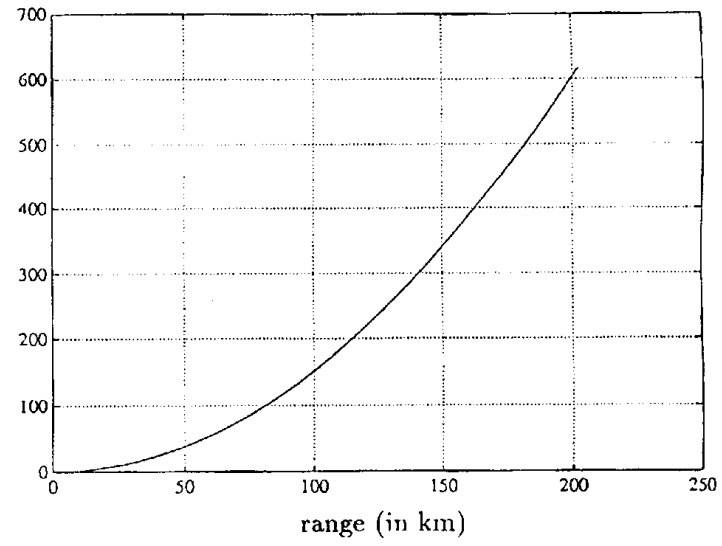

Fig. 3. Values of $2 r_{3}$ (in $\mathrm{km}, y$-axis) versus range $r$ ( $x$-axis, in $\mathrm{km}$ ) for source with bearing $\theta=\pi / 4$.

For the rest of this work, the following array parameters have been chosen (frequency $=300 \mathrm{~Hz}$, $d$ : intersensor distance $=\lambda / 2=2.5 \mathrm{~m}$ ).

It may be also worthy considering the following approximation of the directivity function $f(\theta, r)$ (in (4)). This approximation relies on an approximation of the geometric "delays" $h_{j}$ (in (7)). Let us consider

$$
h_{j}=\left(r^{2}+d_{j}^{2}+2 d_{j} r \sin \theta\right)^{1 / 2}-r
$$

with

$$
d_{j}=d\left(\frac{p-1}{2}-j+1\right) \triangleq d a
$$

and $A$ defined as

$$
A=r\left(1+\frac{d^{2} a^{2}}{r^{2}}+2 \frac{d a}{r} \sin \theta\right)^{1 / 2} .
$$

Then, a second-order expansion of the scalar $A$ (wrt: $d^{2} a^{2} / r^{2}+2 d a / r \sin \theta$ ) is

$$
\begin{aligned}
A \stackrel{2}{=} r & {\left[1+\frac{1}{2}\left(\frac{d^{2} a^{2}}{r^{2}}+2 \frac{d a}{r} \sin \theta\right)\right.} \\
& \left.-\frac{1}{8}\left(\frac{d^{2} a^{2}}{r^{2}}+2 \frac{d a}{r} \sin \theta\right)^{2}\right] .
\end{aligned}
$$

In the above expression, the terms $d^{4} a^{4} / r^{4}$ and $4 d^{3} a^{3} \sin \theta / r^{3}$ may be neglected, providing thus the following approximation of $A$ (acceptable in the Fresnel zone):

$$
A-r \simeq d a \sin \theta+\frac{1}{2} \frac{d^{2} a^{2}}{r} \cos ^{2} \theta
$$

and finally:

$$
\begin{aligned}
f(\theta, r)=\mid \Sigma_{a} \exp \{2 i \pi f / c[ & d a\left(\sin \theta_{0}-\sin \theta\right)+\frac{d^{2} a^{2} \cos ^{2} \theta_{0}}{2 r_{0}} \\
& \left.\left.-\frac{d^{2} a^{2} \cos ^{2} \theta}{2 r}\right]\right\}\left.\right|^{2}
\end{aligned}
$$

The above expression shows that $f(\theta, r)$ is approximately composed of two terms: an angular term corresponding to $d a\left(\sin \theta-\sin \theta_{0}\right)$ and an angular-range term associated with the elementary factors $\left(d^{2} a^{2} \cos ^{2} \theta_{0} / 2 r_{0}-d^{2} a^{2} \cos ^{2} \theta / 2 r\right)$.

Very roughly speaking, it seems thus that the range beamwidth $2 r_{3}$ is a function of $1 / r_{0}$. Another interesting consequence of this approximation is that a convenient reparametrization of the problem may consist in replacing the range $r$ by its inverse $1 / r$.

Assume now that $\theta=\theta_{0}$, then it comes

$$
f\left(\theta_{0}, r\right)=\left|\Sigma_{a} \exp \left\{i \pi \frac{f d}{c}\left(\cos ^{2} \theta_{0}\right) a^{2}\left(1 / r-1 / r_{0}\right)\right\}\right|^{2} .
$$

Clearly, the maximum of the function $f\left(\theta_{0}, r\right)$ is attained for $r=r_{0}$. However, note the difference with the classical Fejer kernel (of the classical beamforming) due to the $a^{2}$ terms. Thus the generalized ambiguity function (GAF) $f(\theta, r)$ has two different structures [3]. Along the $\Delta_{1}=\Delta \sin \theta$ axis the GAF has the sinc-squared structure, while along the radial $\Delta\left(\cos ^{2} \theta / r_{0}\right)$ axis it has the Fresnel structure [3], i.e., with the notations of [3]:

$$
f\left(r_{0}, r\right) \sim|F(\Sigma) / \Sigma|^{2}
$$

with

$$
F(\Sigma)=\int_{0}^{\Sigma} \exp \left(i t^{2}\right) d t=\text { Fresnel exponential integral }
$$

and

$$
\Sigma=\left[\pi / \lambda \Delta\left(\cos ^{2} \theta_{0} / r_{0}\right)\right]^{1 / 2} L / 2, \quad L=p d .
$$

This approximation is furthermore quite enlightening for the analysis of the interference (between sources) phenomenon.

Assume that two sources are present, then

$$
f(\theta, r)=f_{1}(\theta, r)+f_{2}(\theta, r)+f_{12}(\theta, r)
$$

where $f_{1}(\theta, r)$ is the directivity function associated with the source 1 , idem for $f_{2}, f_{12}(\theta, r)$ is the interference term, and direct calculations provide the following approximation of $f_{12}(\theta, r)$ :

$$
\begin{aligned}
f_{12}(\theta, r)=2 \sum_{a, b} \cos \left\{\frac{\pi f}{c}[\right. & d^{2} a^{2}\left(\frac{1}{r_{1}}-\frac{1}{r}\right) \\
+ & \left.\left.d^{2} b^{2}\left(\frac{1}{r}-\frac{1}{r_{2}}\right)\right]\right\}
\end{aligned}
$$

( $r_{1}$ : source 1 range, idem for $r_{2} ; \theta_{1,0}=\theta_{2,0}=0$ ).

This term explains the strong interferences between sources.

These deterministic calculations must be completed by the calculation of the Cramer-Rao bounds (CRB for the sequel) relatively to $r$ and $\theta$. In the case of 
a fixed source the calculations are quite classical $[2$, 4], the elements of the Fisher Information Matrix $F$ (FIM) are given below (Bang's formula) [5]:

with

$$
F\left(\theta_{1}, \theta_{2}\right)=-\operatorname{tr}\left[\frac{\partial R}{\partial \theta_{1}} R^{-1} \frac{\partial R}{\partial \theta_{2}} R^{-1}\right]
$$

$R$ is the covariance matrix of the observations $X$ (array output)

$R=\sigma D_{\theta, r} D_{\theta, r}^{*}+b I d$

$\rho=\sigma / b$ signal-to-noise ratio

tr is the trace of a matrix

$\theta_{1}=\theta$ or $r, \theta_{2}=\theta$ or $r$.

It is worth comparing these (experimental) results with the theoretical results of the literature $[2,4,6]$. Using the approximation (12), L. Kopp and D. Thubert [2] have obtained a very simple and interesting form of the FIM relatively to the parameters $\theta_{1}=\sin \theta$ and $\theta_{2}=r^{-1}$, i.e.,

$$
F\left(\theta_{1}, \theta_{2}\right)=\frac{2 \rho^{2} w^{2}}{1+p \rho} \operatorname{diag}\left(\frac{\alpha^{2} p^{2}\left(p^{2}-1\right)}{12}, \frac{\beta^{2} p^{2}\left(p^{2}-1\right)\left(p^{2}-4\right)}{180}\right)
$$

with

$$
\begin{array}{ll}
\omega=2 \pi f, & \rho=\frac{s}{b}, \quad \alpha=\frac{d}{c}, \quad \beta=\frac{d^{2} \cos ^{2} \theta}{2 c} \\
\theta_{1}=\sin \theta, & \theta_{2}=r^{-1}, \quad \text { diag: diagonal matrix. }
\end{array}
$$

The diagonal form of the approximated (17) FIM is remarkable. This can also been considered as a consequence of the orientation of the uncertainty ellipsoid (with axis $2 \theta_{3}$ and $2 r_{3}$, in (9)). Furthermore, it is worth noting the general form of the $3 \times 3$ Fisher matrix $F(\rho, \theta, r)$ :

$$
F(\rho, \theta, r)=\left(\begin{array}{ccc}
f(\rho, \rho) & 0 & 0 \\
0 & & \\
& F(\theta, r) \\
0 & &
\end{array}\right)
$$

$(f(\rho, \rho)$ is a scalar).

Therefore the analysis is restricted to $F(\theta, r)$ for the rest of this work.

The error bounds relative to the estimation of $\theta_{1}$ and $\theta_{2}$ are directly deduced from (16) and stands as follows [2]:

$$
\begin{aligned}
& \sigma_{\sin \theta}^{C R}=\theta_{3} \frac{\sqrt{1+p \rho}}{p \rho}\left(\theta_{3} \text { given by }(5)\right) \\
& \sigma_{r-1}^{C R}=\left(\frac{(1+p \rho) 360 c^{2}}{\rho^{2} \omega^{2}\left(d^{2} \cos ^{2} \theta\right)^{2} p^{2}\left(p^{2}-1\right)\left(p^{2}-4\right)}\right)^{1 / 2}
\end{aligned}
$$

(standard deviation (SD) bounds).

It is interesting to rewrite the error bound $\sigma_{r^{-1}}^{C R}$ in the following form:

$$
\sigma_{r-1}^{C R}=\alpha_{3} \frac{\sqrt{1+p \rho}}{p \rho}
$$

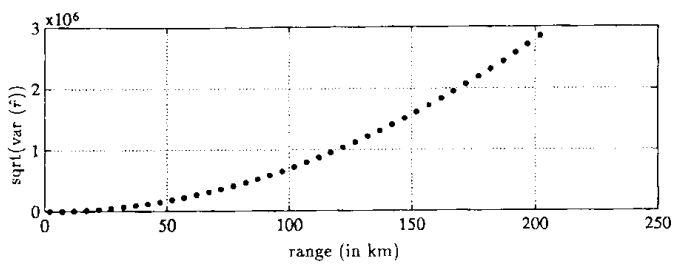

Fig. 4. Variance (sqrt) of the estimated range (in $\mathrm{m}, y$-axis) versus range (in $\mathrm{km}, x$-axis) for fixed source and linear array $(p=100$, $d=\lambda / 2, \theta=\pi / 4$ ).

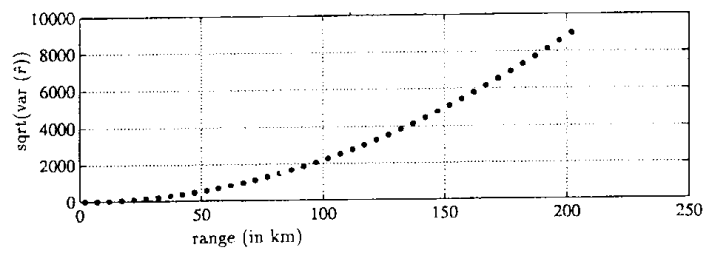

Fig. 5. Variance(sqrt) of estimated range (in $\mathrm{m}, y$-axis) versus range (in $\mathrm{km}, x$-axis) for fixed source and linear array ( $p=1000$, $d=\lambda / 2, \theta=\pi / 4)$.

with

$$
\begin{aligned}
2 \alpha_{3} & =\frac{6 \sqrt{10}}{\pi} \frac{\lambda}{L^{2} \cos ^{2} \theta}\left(\frac{(p-1)^{3}}{(p+1)\left(p^{2}-4\right)}\right) \\
L & =p d \quad p \geq 3 .
\end{aligned}
$$

The term $2 \alpha_{3}$ can thus be considered as an inverse range half-beamwidth. Note that it doesn't depend of $r$ and that the following relations hold from $(18,19)[2]:$

$$
\frac{\sigma_{\sin \theta}^{C R}}{\sigma_{r^{-1}}^{C R}}=\frac{\theta_{3}}{\alpha_{3}} \sim \frac{L \cos ^{2} \theta}{\sqrt{60}} \quad(p \gg 1) .
$$

If now we choose $\theta=0$, then it comes

$$
\frac{\sigma_{r}^{C R}}{r}=r \sigma_{r^{-1}}^{C R} \simeq \frac{r}{L} \sqrt{60} \sigma_{\sin \theta}^{C R} .
$$

This approximation is important since it proves that the relative error for estimating the range $r$ is directly related to the variance of the bearing estimate in the one hand and to the ratio $r / L$ in the other.

It is worth noting that the formula (16) corresponds to the case where the observation is reduced to a single snapshot $(N=1)$. In the general case, the elements of $F$ are multiplied by the scalar $N$ and thus the lower bounds of the estimator variance are divided by $N$.

For the sake of computation cost, (16) may be expanded in order to require only the computation of scalar products, this detail having a considerable importance for a large array (e.g., $p=1000$ ). A lower bound of the variance of $\hat{r}$ is deduced from (16). This calculation is illustrated by Figs. 4 and 5 . For the two figures the source parameters are identical $(\rho=\sigma / b=$ $0 \mathrm{~dB}, \theta=\pi / 4$, fixed sources), $N$ the snapshot number is equal to 1 (for the both figures) while the sensor number $p$ is equal to 100 for Fig. 4 and to 1000 for Fig. 5, the intersensor distance $d$ being equal to $\lambda / 2$. 


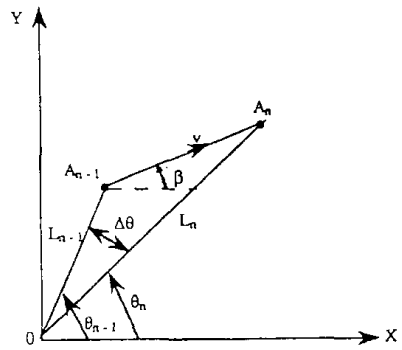

Fig. 6. Geometry of source motion ( $A_{n}$ and $A_{n-1}$ are source position at time $t_{n}$ and $t_{n-1}$, respectively).

The graph represents the square root of $\operatorname{var}(\hat{r})$ (SD $(\hat{r})$ in meters) versus the distance $d$ of the source (in kilometers, $x$-axis). As expected, the SD of $\hat{r}$ is considerably reduced as $p$ increases from 100 to 1000 since it is approximately divided by a factor 500 .

Actually, this (experimental) result may also be illustrated by a formula established by Schultheiss in the wideband case $(N=1)[4]$ :

$$
\begin{aligned}
\operatorname{var}(\hat{\theta}) & \geq 6 \frac{c^{2}}{K} \frac{\frac{p-1}{p+1}}{L^{2} \sin ^{2} \theta} \\
\operatorname{var}(\hat{r}) & \geq \frac{360 c^{2}}{K} \frac{r^{4}}{L^{4} \sin ^{4} \theta} \cdot \frac{(p-1)^{3}}{(p+1)\left(p^{2}-4\right)} \\
\text { with } K & =\frac{T}{2 \pi} \int_{0}^{\infty} \frac{\omega^{2} \cdot(p S(\omega) / B(\omega))^{2}}{1+p S(\omega) / B(\omega)} \\
\text { and } \omega & =2 \pi f \quad L=p d
\end{aligned}
$$

$\theta$ referenced wrt the array broadside.

This formula shows that $\operatorname{var}(\hat{r})$ depends of the range as a factor of $(r / L)^{4}$ and agrees with the results of J. M. F. Moura and A. B. Baggeroer [3].

\section{ON THE ESTIMATION OF THE RANGE OF A MOVING SOURCE}

For the sequel, the following assumptions are made (for the sake of simplicity): the source motion is linear and uniform (the velocity vector is constant), the array is fixed, the working temporal frequency is omitted.

We now consider the effects of source motion on its estimated range $[7,8]$. The classical beamforming (CBF) consists in calculating the following quadratic form with the notations of Section II.

$$
\begin{aligned}
& \hat{P}(\theta, r)=\frac{1}{N} \sum_{t=0}^{N-1}\left|X_{t}^{*} D_{\theta, r}\right|^{2} \\
&=D_{\theta, r}^{*}\left(\frac{1}{N} \sum_{t=0}^{N-1} X_{t} X_{t}^{*}\right) D_{\theta, r} \triangleq P_{\theta, r}(\hat{R}) \\
& \text { with } X_{t}: \text { snapshot vector }[1] \\
& \text { then } \quad(\hat{\theta}, \hat{r}) \triangleq \arg \max g(\theta, r) .
\end{aligned}
$$

Since we are concerned with the effects of the source motion on the array processing performance, a simplified model of the range is considered. More precisely, let $r_{n}$ be the source array distance at the time $t_{n}\left(t_{n}=n \Delta T, \Delta T\right.$ : snapshot time) then (with the notations of Fig. 6) the following relations are easily deduced from classical trigonometry:

$$
\mid \begin{aligned}
& r_{n} \cos \theta_{n}=r_{0} \cos \theta_{0}+(\cos \beta) n v \Delta T \\
& \text { (equality of the projections) } \\
& r_{n}^{2}=r_{0}^{2}+(n v \Delta T)^{2}+2 r_{0}(n v \Delta T) \cos \left(\theta_{0}-\beta\right) \\
& \text { (norm equality) }
\end{aligned}
$$$$
\text { ( } \beta \text { : source heading). }
$$

Assuming that the factor $x\left(x=n v \Delta T / r_{0}\right)$ is little in regard to 1 , first- order expansions of the range $r_{n}$ and $\cos \theta_{n}$ are directly deduced from (22), i.c.,

$$
\begin{gathered}
r_{n} \stackrel{1}{=} r_{0}+\cos \left(\theta_{0}-\beta\right)(n v \Delta T) \\
\cos \theta_{n} \stackrel{1}{=} \cos \theta_{0}+\left[\cos \beta-\cos \theta_{0} \cos \left(\theta_{0}-\beta\right)\right]\left(\frac{n v \Delta T}{r_{0}}\right)
\end{gathered}
$$

(the symbol $\stackrel{1}{=}$ meaning 1 st order expansion).

If $N$ snapshots are considered (in (21)) the mean values $\left(\cos \theta_{M}, r_{M}\right)$ of $\cos \theta_{n}$ and $r_{n}$ are thus approximated at the first order:

$$
\begin{aligned}
\cos \theta_{M} \stackrel{1}{=} & \cos \theta_{0} \\
& +\left[\frac{\left(\cos \beta-\cos \theta_{0} \cos \left(\theta_{0}-\beta\right)\right) v \Delta T}{r_{0}} \times\left(\frac{N-1}{2}\right)\right] \\
r_{M} \stackrel{1}{=} & r_{0}+\cos \left(\theta_{0}-\beta\right) v \Delta T \cdot\left(\frac{N-1}{2}\right)
\end{aligned}
$$

The source motion results in a spreading of the source mainlobe wrt the parameters $\theta$ and $r$. Contrary to the stationary case (i.e., fixed sources) it is not possible to calculate the CRB (wrt $\theta$ and $r$ in the case of a moving source (it may be considered as Bayesian problem); however the variance of the (averaged) estimators of $r_{M}$ (i.e., $\hat{r}_{M}$ ) and $\theta_{M}$ (i.e., $\hat{\theta}_{M}$ ) can be calculated by using a Taylor expansion on a neighborhood of $r_{M}$ and $\theta_{M}$.

For that purpose, consider the derivative $g(\Omega, \hat{r})$ wrt the parameter vector $\Omega\left(\Omega \triangleq(\theta, r)^{t}\right)$ of $P(\Omega, \hat{R})(21)$ defined below:

$$
\begin{aligned}
\hat{R} & =\frac{1}{N} \sum_{t=0}^{N-1} X_{t} X_{t}^{*}\left(R_{t} \triangleq \operatorname{cov}\left(X_{t} X_{t}^{*}\right), R \triangleq \frac{1}{N} \sum_{t=0}^{N-1} R_{t}\right) \\
g(\Omega, \hat{R}) \triangleq & \triangleq \begin{array}{l}
\frac{\partial}{\partial \theta} P(\Omega, \hat{R}) \\
\frac{\partial}{\partial r} P(\Omega, \hat{R})
\end{array}=\nabla P(\Omega, \hat{R}) .
\end{aligned}
$$


Then a first-order expansion of $g\left(\hat{\Omega}_{M}, \hat{R}\right)$, is considered, i.e.,

$$
g\left(\hat{\Omega}_{M}, \hat{R}\right) \stackrel{1}{=} g\left(\Omega_{M}, \hat{R}\right)+\left(\nabla g\left(\Omega_{M}, \hat{R}\right)\right)\left(\hat{\Omega}_{M}-\Omega_{M}\right)
$$

itself equivalent to

$$
\begin{aligned}
g\left(\hat{\Omega}_{M}, \hat{R}\right) \simeq & g\left(\Omega_{M}, \hat{R}\right) \\
& +\left(\nabla g\left(\Omega_{M}, R\right)+\nabla g\left(\Omega_{M}, \hat{R}-R\right)\right)\left(\hat{\Omega}_{M}-\Omega_{M}\right)
\end{aligned}
$$

so that finally, neglecting the second-order term (i.e., $\nabla g(\Omega, \hat{R}-R))$, the following linear approximation of $g\left(\hat{\Omega}_{M}, \hat{R}\right)$ is obtained:

$$
g\left(\hat{\Omega}_{M}, \hat{R}\right) \simeq g\left(\Omega_{M}, \hat{R}\right)+\left(\nabla g\left(\Omega_{M}, R\right)\right)\left(\hat{\Omega}_{M}-\Omega_{M}\right) .
$$

Since, by definition of $\hat{\Omega}_{M}$, the nullity of $g\left(\hat{\Omega}_{M}, \hat{R}\right)$ is assumed, the following equality is straightforwardly deduced from (26):

$$
\hat{\Omega}_{M}-\Omega_{M} \simeq-\left(\nabla g\left(\Omega_{M}, R\right)\right)^{-1} g\left(\Omega_{M}, \hat{R}\right) .
$$

Using (27), it is possible to derive an estimation of the variances of $\hat{\theta}_{M}$ and $\hat{r}_{M}$ (respectively $\operatorname{var}\left(\hat{\theta}_{M}\right)$ and $\operatorname{var}\left(\hat{r}_{M}\right)$ ), given by (Appendix A and [7]):

$$
\operatorname{var}\left(\hat{r}_{M}\right)=\frac{1}{N^{2}} \frac{\sum_{t=0}^{N-1} \operatorname{tr}\left[\left(A R_{t}\right)^{2}\right]+\left(\sum_{t=0}^{N-1} \operatorname{tr}\left(A R_{t}\right)\right)^{2}}{\left[\operatorname{Re}\left(V_{r_{M}}^{*} R D_{\Omega_{M}}\right)+U_{r_{M}}^{*} R U_{r_{M}}\right]^{2}}
$$

with

$$
\begin{aligned}
U_{r_{M}} & \triangleq\left(\frac{\partial}{\partial r} D_{\theta, r}\right)_{r=r_{M}} ; \quad V_{r_{M}} \triangleq\left(\frac{\partial^{2}}{\partial r^{2}} D_{\theta, r}\right)_{r=r_{M}} \\
A & =\frac{1}{2}\left(C+C^{*}\right) \quad \text { with } \quad C=U_{r_{M}} \cdot D_{\Omega_{M}}^{*} .
\end{aligned}
$$

Note that (28) has been obtained by means of a first-order expansion of the functional $g(\Omega, \hat{R})$ and at the expense of the approximation (26). This approach has the advantage of simplicity but can be replaced by the more rigourous approach of $D$. F. Gingras and S. L. Hobbs $[9,10]$. More precisely, let $\Omega(R)$ the value of the parameter vector $\Omega$ defined (implicitely) by

$$
g(\Omega(R), R)=0
$$

Thanks to the implicit function theorem [11], $\Omega(R)$ is a differentiable function of the matrix, and its "partial" derivative wrt $R$ is given by (differentiation chain rule) [11]:

$$
\frac{\partial}{\partial R} g(\Omega(R), R)+\frac{\partial g}{\partial \Omega}(\Omega(R), R) \cdot \frac{\partial \Omega(R)}{\partial R} .
$$

Now, by definition of $\Omega(R)$, the above partial derivative is null, so that

$$
\frac{\partial \Omega(R)}{\partial R}=-\left(\frac{\partial g}{\partial \Omega}(\Omega(R), R)\right)^{-1} \frac{\partial g}{\partial R}(\Omega(R), R) .
$$

The rest of the derivation relies on two classical lemmas.
LEMMA 1 [12]

$$
\lim _{N \rightarrow \infty} \operatorname{cov}\left(\tilde{r}_{i j}, \tilde{r}_{k \ell}\right)=\frac{1}{N} r_{i k} r_{j \ell}
$$

with

$$
\begin{aligned}
\tilde{r}_{i j} & \triangleq \hat{r}_{i j}-r_{i j} \\
r_{i j} & =\text { element of } R, i \text { th row, } j \text { th column } \\
\mathbb{E}\left(\tilde{r}_{i j}\right) & =r_{i j} .
\end{aligned}
$$

The elements of the matrix $\hat{R}$ can be (asymptotically) considered as a complex circular [13] Gaussian vector with mean and covariance given above.

LEMMA 2 [10, 12] Assume that $Z$ is asymptotically $a$ gaussian vector of mean $Z_{0}$ and covariance $(1 / N) \Sigma$, and $f$ an analytical vector-valued function of $Z$, then the vector $f(Z)$ is itself asymptotically gaussian with mean $f\left(Z_{0}\right)$ and covariance $(1 / N) f^{\prime} \Sigma f^{\prime *}\left(f^{\prime}=\partial f / \partial Z\right)$.

Applying Lemmas 1 and 2 , the vector $\Omega(\hat{R})$ is asymptotically Gaussian and its covariance stands as follows:

$$
\operatorname{cov}(\Omega(\hat{R}))=\left(\frac{\partial \Omega(R)}{\partial R}\right) \operatorname{cov}(\hat{R})\left(\frac{\partial \Omega(R)}{\partial R}\right)^{*}
$$

$\partial \Omega(R) / \partial R$ given by (29) so that, finally

$$
\operatorname{cov}(\Omega(\hat{R}))=\frac{1}{N}\left(\frac{\partial g(\Omega, R)}{\partial \Omega}\right)^{-1} M\left(\frac{\partial g(\Omega, R}{\partial \Omega}\right)^{-1 *}
$$

with

$$
\begin{array}{r}
M_{m, n}=\left(\sum_{i, j, k, \ell} \frac{\partial g}{\partial r_{i j}} \frac{\partial g}{\partial r_{k \ell}} r_{i k} r_{\ell j}\right)_{m, n} \\
(m \leftarrow \theta, n \leftarrow r) .
\end{array}
$$

Here, the function $g$ is the partial derivative (wrt $\Omega$ ) of the function $P\left(\Omega_{M}, R\right)$, the averaged parameter vector $\Omega_{M}$ being defined by (24) and $R$ being the exact averaged matrix (i.e., $R=(1 / N) \sum_{t=0}^{N}\left(R_{t}\right)$ ).

The approach of S. L. Hobbs [10] seems more general than the classical method of expansion (26). However, the computational burden is bigger (this is not very important) and, overall, the results of the two approaches are rather similar.

The effect of the temporal averaging on the variance of the estimated range is illustrated by Figs. 7 to 13 .

A "short" array is considered for Figs. 7-9. In the first case, the source parameters are $\theta_{0}=2 \pi / 3$ and $\beta=\pi / 4, \nu=10 \mathrm{~m} / \mathrm{s}, r_{0}=10 \mathrm{~km}, \rho=-10 \mathrm{~dB}$, and $p$, the number of sensors, is 50 .

The SD of $\hat{r}$ is approximated by (28), actually Monte-Carlo runs have shown that this approximation is quite accurate. The SD of $\hat{r}$ is plotted on the $y$-axis versus $N$ (on the abscissa). The minimal value of $\mathrm{SD}$ $(\hat{r})$ is attained for $N \simeq 60$, note that this value is rather 


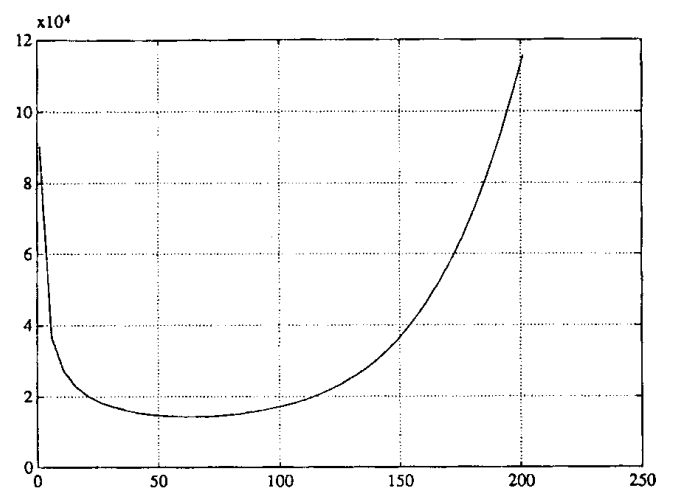

Fig. 7. Variance (sqrt) of the estimated range (in $\mathrm{m}, y$-axis) versus $N$ (number of snapshots, $\Delta T=1 \mathrm{~s}$ ) for a close moving source $\left(\theta_{0}=2 \pi / 3, \beta=\pi / 4, v=10 \mathrm{~m} / \mathrm{s}, r_{0}=10 \mathrm{~km}, \rho=-10 \mathrm{~dB}\right)$ and a short array $(p=50, d=\lambda / 2)$

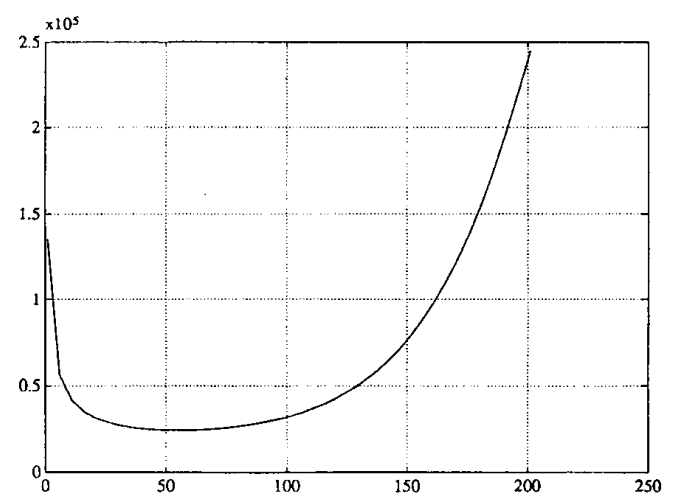

Fig. 8. Variance (sqrt) of the estimated range (in $\mathrm{m}, y$-axis) versus $N$ (number of snapshots, $\Delta T=1 \mathrm{~s}$ ) for a close moving source $\left(\theta_{0}=\pi / 4, \beta=\pi / 4, v=10 \mathrm{~m} / \mathrm{s}, r_{0}=10 \mathrm{~km}, \rho=-10 \mathrm{~dB}\right)$ and $\mathrm{a}$ short array $(p=50, d=\lambda / 2)$.

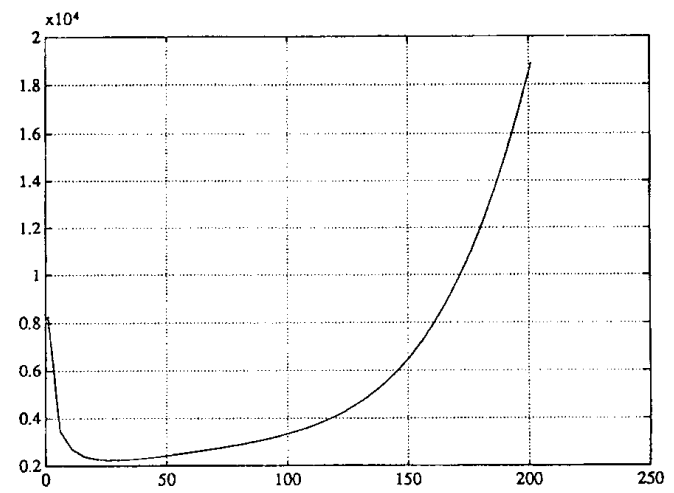

Fig. 9. Variance (sqrt) of the estimated range (in $m, y$-axis) versus $N$ (number of snapshots, $\Delta T=1 \mathrm{~s}$ ) for a close moving source $\left(\theta_{0}=2 \pi / 3, \beta=\pi / 4, v=10 \mathrm{~m} / \mathrm{s}, r_{0}=10 \mathrm{~km}, \rho=10 \mathrm{~dB}\right)$ and a short array $(p=50, d=\lambda / 2)$.

important and that $\operatorname{var}(\hat{r})$ increases quickly with $N$ (for $N \leq 150$ ).

The behavior of SD $(\hat{r})$ is similar for other choices of sources parameters (Figs. 8 and 9). For Fig. 8, the

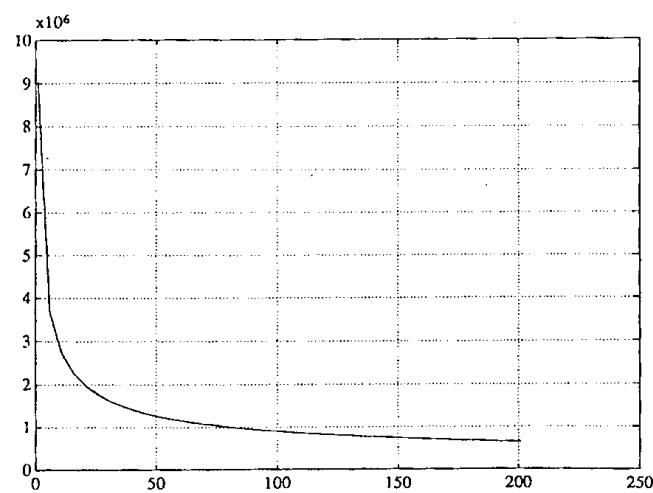

Fig. 10. Variance (sqrt) of estimated range (in $\mathrm{m}, y$-axis) versus $N$ for a far moving source $\left(\theta_{0}=\pi / 4, \beta=\pi / 4, v=10 \mathrm{~m} / \mathrm{s}\right.$, $\left.r_{0}=100 \mathrm{~km}, \rho=-10 \mathrm{~dB}\right)$ and short array $(p=50, d=\lambda / 2)$.

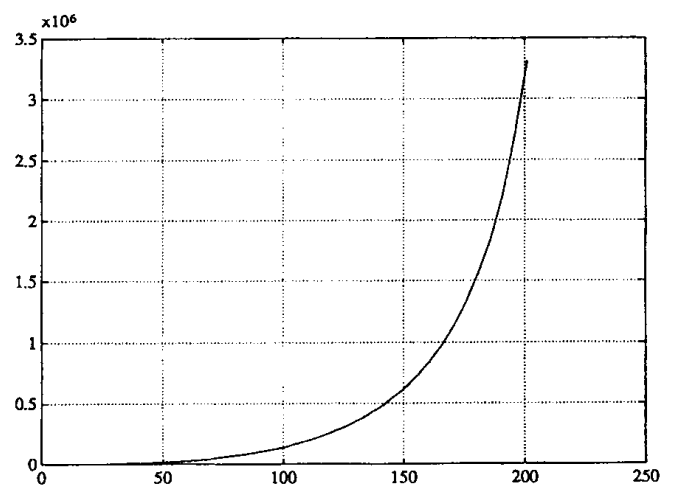

Fig. 11. Variance (sqrt) of estimated range (in $\mathrm{m}, y$-axis) versus $N$ for close moving source $\left(\theta_{0}=2 \pi / 3, \beta=\pi / 4, v=10 \mathrm{~m} / \mathrm{s}\right.$, $\left.r_{0}=10 \mathrm{~km}, \rho=-10 \mathrm{~dB}\right)$ and large array $(p=500, d=\lambda / 2)$.

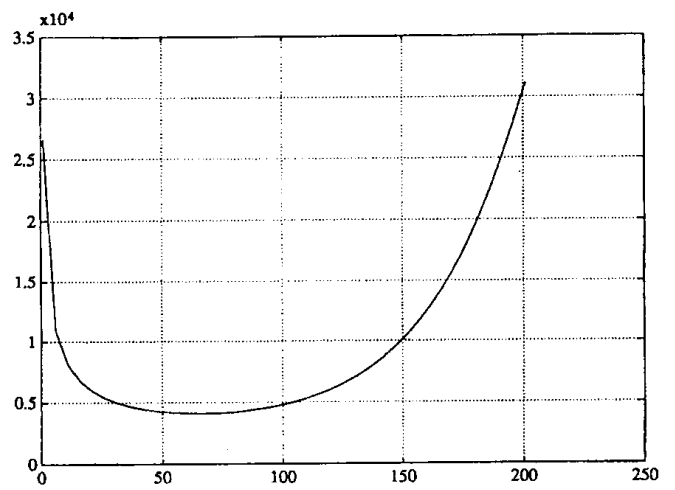

Fig. 12. Variance (sqrt) of estimated range (in $\mathrm{m}, y$-axis) versus $N$ for far moving source $\left(\theta_{0}=2 \pi / 3, \beta=\pi / 4, \nu=10 \mathrm{~m} / \mathrm{s}\right.$, $\left.r_{0}=10 \mathrm{~km}, \rho=-10 \mathrm{~dB}\right)$ and large array $(p=500, d=\lambda / 2)$.

source parameters are $\theta_{0}=\pi / 4$, and $\beta=\pi / 4$, the other parameters are unchanged. Then, the values of SD $(\hat{r})$ are modified but not its behavior (wrt $N$ ). The case of a strong source $(\rho=10 \mathrm{~dB})$ is considered in Fig. 9, the source parameters are those of Fig. 7. The minimum 


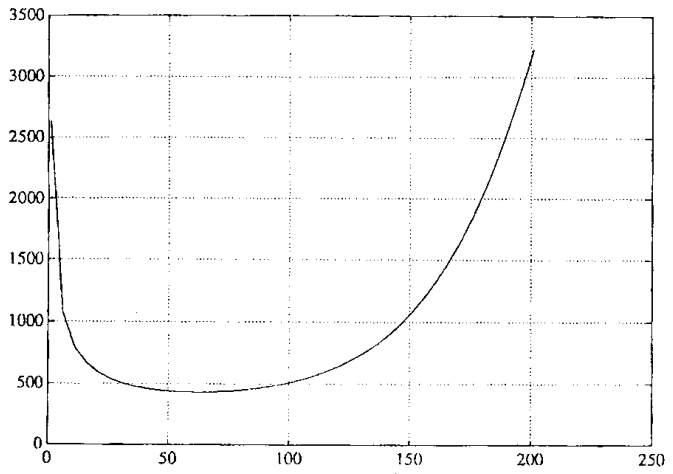

Fig. 13. Variance (sqrt) of estimated range (in $\mathrm{m}, y$-axis) versus $N$ for far and strong moving source $\left(\theta_{0}=2 \pi / 3, \beta=\pi / 4\right.$, $v=10 \mathrm{~m} / \mathrm{s}, \rho=+10 \mathrm{~dB})$ and large array $(p=500, d=\lambda / 2)$.

of SD $(\hat{r})$ is sharpened and the values (of SD $(\hat{r})$ ) are divided by a factor which is approximately equal to 10. This factor agrees with the theoretical calculation of $\operatorname{var}(\hat{r})$ which asserts that $\operatorname{var}(\hat{r})$ depends on $\rho$ as a factor of $(1 / \rho)^{1 / 2}$ (exactly equation $\left.(1+p \rho)^{1 / 2} / p \rho\right)$ [4].

On the opposite, the behavior of $\operatorname{SD}(\hat{r})$ is completely modified by the initial source distance $r_{0}$. Thus, it is equal to $100 \mathrm{~km}$ for Fig. 10; the other source and array parameters are identical to those of Fig. 8. Then, the effect of the choice of $N$ is less dramatic, but the values of $\mathrm{SD}(\hat{r})$ are rather important. These different behaviors of SD $(\hat{r})$ (versus $r_{0}$ ) can be easily explained by the size of the resolution cells i.e., $2 \theta_{3}$ and $2 r_{3}$ which are much larger for a far source (see Fig. 18).

Another important factor is $p$. Its effects are illustrated on Figs. 11, 12, and 13. A low range source is considered for Fig. 11 as well as a very large sensor number $(p=500)$, and the source parameters are those of Fig. 7 (i.c., $r_{0}=10 \mathrm{~km}, v=10 \mathrm{~m} / \mathrm{s}, \rho=$ $\left.-10 \mathrm{~dB}, \theta_{0}=2 \pi / 3, \beta=\pi / 4\right)$. Then, the optimum value of $N$ is very little and the array performance in distance estimation is deeply, degraded by increasing $N$. For a long range source $\left(r_{0}=100 \mathrm{~km}\right)$, the behavior of SD $(\hat{r})$ is illustrated by Figs. 12 and 13 . They are quite similar to those obtained for $p=$ 50 and $r_{0}=10 \mathrm{~km}$, the behavior of SD $(\hat{r})$ seems therefore very depending on the ratio $r_{0} / L(L=p d$, array length). Note that the minimum value of $\mathrm{SD}(\hat{r})$ for a strong source $\rho=10 \mathrm{~dB}$ and a distant source is rather low $(p=500)$ since it is approximately $500 \mathrm{~m}$.

\section{RANGE ESTIMATION BY USING A PARTITIONED ARRAY}

As previously, the array is assumed to be linear and constituted of $p$ equispaced sensors. We now consider that the great array is partitioned into $\ell$ subarrays, each of them corresponding to an array of $p / \ell$ sensors.

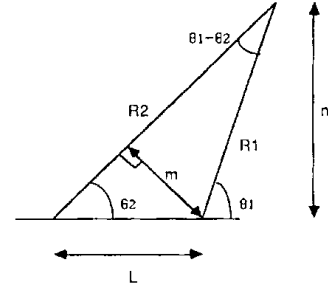

Fig. 14. Definition of triangulation parameters.

Actually, this approach for range estimation can be seriously motivated as it will be seen. One reason is the computation cost of a focused beamforming (4), (20) for a large array which may become tremendous. Another one is perhaps even more fundamental, the optimal integration time (i.e., $N \Delta T$ ) for range estimation may be very low (as it has been seen previously in Section III) and depend highly on the source trajectory parameters which are generally unknown. Other reasons are the wavefront spatial coherence [1] and the effects of bias in estimating the cross-spectral matrix $\hat{R}[14]$. Therefore, partitioning the array seems to be a good mean for "robustifying" the focused beamforming. Note that this partition can also be considered as an approximation of the circular wavefront by a regular polygon. This simple constatation is instrumental. The performance of a two-arrays system is well known $[15,16]$ but this is not true for a more general $\ell$-arrays system in the presence of moving sources.

This is the major aim of the Section IV. Classical results for two arrays (triangulation) are briefly presented and then the performance analysis is extended to more general systems.

\section{A. Triangulation Performance}

The array is divided into two equal arrays (each of $p / 2$ sensors, $p$ even). On each subarray, the plane wave assumption is made (even if it is not very realistic) and each subarray two bearings $\theta_{1}$ and $\theta_{2}$ are estimated as depicted in Fig. 14. With the notations of Fig. 14, one has

$$
m=r_{1} \sin \left(\theta_{1}-\theta_{2}\right)=L \sin \theta_{2}
$$

( $L$ is the distance between the acoustical centers of the two subarrays) and thus

$$
r_{1}=L \frac{\sin \theta_{2}}{\sin \left(\theta_{1}-\theta_{2}\right)}
$$

Similarly, one has

$$
n=r_{1} \sin \left(\theta_{1}\right)=r_{2} \sin \left(\theta_{2}\right)
$$

and therefore

$$
r_{2}=L \frac{\sin \theta_{1}}{\sin \left(\theta_{1}-\theta_{2}\right)}
$$


An estimator of $r_{1}$ is obtained by replacing the exact bearings $\theta_{1}$ and $\theta_{2}$ into (33) or (34) by their respective estimates, i.e.,

$$
\hat{r}_{1}=L \frac{\sin \hat{\theta}_{2}}{\sin \left(\hat{\theta}_{1}-\hat{\theta}_{2}\right)} .
$$

The term $\hat{r}_{1}$ defined by (35) is an estimator of $r_{1}$ (triangulation). The statistical performance of this estimator have been carefully studied for a fixed source $[15,16]$ leading thus to the following results:

$$
\begin{aligned}
& \operatorname{MSE}\left(\hat{r}_{1}\right) \simeq \sigma_{\theta}^{2} \frac{2 r^{4}}{L^{2} \sin ^{2} \theta} \\
& \operatorname{bias}\left(\hat{r}_{1}\right) \simeq 2 \sigma_{\theta}^{2} \frac{r^{3}}{L^{2} \sin ^{2} \theta} \quad\left(r_{1} \simeq r_{2}, \theta_{1} \simeq \theta_{2}\right)
\end{aligned}
$$

where MSE is mean square error.

In the formula (22) $\sigma_{\theta}^{2}$ represents the variance of the estimated bearing itself given by the Woodward formula, i.e.,

$$
\begin{aligned}
\sigma_{\theta} & =\frac{1}{\sqrt{N}} \theta_{3} \frac{\sqrt{1+q \rho}}{q \rho} \\
2 \theta_{3} & =\frac{\sqrt{6}}{\pi} \frac{\lambda}{L} \sqrt{\frac{q-1}{q+1}} \cdot \frac{1}{\sin \theta}
\end{aligned}
$$

with

$$
\begin{aligned}
& L=q d, \quad q=p / 2 \\
& d: \text { intersensor distance } \\
& \rho: \text { signal/noise ratio }
\end{aligned}
$$

0 deg: array axis.

For the sake of clarity, a proof of the classical formulas (36) is briefly presented in Appendix B.

B. On the Performance of Partitioned Processing for Range Estimation

This time, the array is divided into $\ell$ subarrays (each of $p / \ell$ sensors). As previously, the plane wave assumption is made on each subarray. A sequence of $\ell$ estimated bearings $\left\{\hat{\theta}_{1}, \hat{\theta}_{2}, \ldots, \hat{\theta}_{\ell}\right\}$ is thus associated with each source.

From elementary trigonometry, one has

$$
\sin \theta_{k}=\frac{r_{1}}{r_{k}} \sin \theta_{1} \quad 1 \leq k \leq \ell
$$

with

$$
r_{k}^{2}=r_{1}^{2}+(k-1)^{2} L^{2}+2(k-1) L r_{1} \cos \theta_{1} .
$$

(Note that the angular reference is now the array axis.)

Consider the vector $\hat{X}$ of pseudomeasurements, i.e.,

$$
\hat{X}=L \mid \begin{aligned}
& \sin \hat{\theta}_{2} \\
& \sin \hat{\theta}_{3} \\
& \vdots \\
& \sin \hat{\theta}_{\ell}
\end{aligned}
$$

with

$$
L=q d \quad \text { and } \quad q=p / \ell
$$

and its model

$$
X=L \mid \begin{array}{l|l}
\sin \theta_{2} \\
\sin \theta_{3} \\
\vdots \\
\sin \theta_{\ell}
\end{array}=\begin{aligned}
& r_{1} \sin \left(\theta_{1}-\theta_{2}\right) \\
& r_{2} \sin \left(\theta_{2}-\theta_{3}\right) \\
& r_{\ell-1} \cdot \sin \left(\theta_{\ell-1}-\theta_{\ell}\right) .
\end{aligned}
$$

In (39) the sequence of modeled bearings $\left(\theta_{2}, \theta_{3}, \ldots, \theta_{\ell}\right)$ is defined by (1), (33), and thus one has

$$
\theta_{2}=f_{2}\left(\theta_{1}, r_{1}\right), \ldots, \theta_{\ell}=f_{\ell}\left(\theta_{1}, r_{1}\right) .
$$

A generalization of the triangulation method presented in Section IVA takes the following form:

$$
\begin{aligned}
& \hat{\Omega}=\left(\begin{array}{c}
\hat{r}_{1} \\
\cos \hat{\theta}_{1}
\end{array}\right)=\arg _{\left(\theta_{1}, r_{1}\right)} \min \|X(\Omega)-\hat{X}\|^{2} \\
& \text { with: } \Omega=\left(\begin{array}{c}
r_{1} \\
\cos \theta_{1}
\end{array}\right) .
\end{aligned}
$$

Note that the vector of source parameters has been slightly modified wrt (2) for the sake of simplicity. The calculation of the variance of $\hat{\Omega}$ is conducted in the same way as previously. More precisely let $f$ be the functional defined by (39), (41), i.e.,

$$
f(\Omega, \hat{X}) \triangleq\|X(\Omega)-\hat{X}\|^{2} .
$$

In the case of a fixed source, then the calculation of the MSE bounds for the bearing and range estimates may be achieved easily. More precisely, consider the following approximations of the vector $X$ components:

$$
\sin \theta_{k}=\frac{r_{1}}{r_{k}} \sin \theta_{1}
$$

with

$$
\begin{aligned}
\frac{r_{1}}{r_{k}} \stackrel{2}{=} & 1-\frac{1}{2}\left(2(k-1) \frac{L}{r_{1}} \cos \theta_{1}+(k-1)^{2} \frac{L^{2}}{r_{1}^{2}}\right) \\
& +\frac{3}{8}\left((k-1)^{2} \frac{L^{2}}{r_{1}^{2}}+2(k-1) \frac{L}{r_{1}} \cos \theta_{1}\right)^{2} .
\end{aligned}
$$

Assuming that $L / r_{1} \ll 1$, the following approximation is finally obtained:

$$
\begin{aligned}
\frac{r_{1}}{r_{k}} \cong & 1-(k-1) \frac{L}{r_{1}} \cos \theta_{1} \\
& +\frac{(k-1)^{2}}{2} \frac{L^{2}}{r_{1}^{2}}\left(1+\cos ^{2} \theta_{1}\right) .
\end{aligned}
$$

LE CADRE: PERFORMANCE ANALYSIS OF WAVEFRONT CURVATURE METHODS 
Furthermore the following assumption is made. The vector $\hat{X}$ is Gaussian i.e.,

$$
\hat{X} \text { is } \mathcal{N}(M, \Gamma)
$$

with

$$
M=X \quad \text { and } \quad \Gamma=L^{2} \cos ^{2} \theta \sigma_{\theta}^{2} I d \triangleq \alpha_{\theta}^{2} I d
$$

( $\sigma_{\theta}^{2}$ given by (37)).

The calculation of the FIM components (relatively to $\theta_{1}$ and $r_{1}$ ) is then direct, leading to

$$
\begin{aligned}
& F_{11} \triangleq-\mathrm{E}\left(\frac{\partial \log p(\hat{X} \mid \Omega)}{\partial \theta^{2}}\right)=\alpha_{\theta}^{-2}\left\|\frac{\partial X}{\partial \theta}\right\|^{2} \\
& F_{22} \triangleq-\mathrm{E}\left(\frac{\partial \log p(\hat{X} \mid \Omega)}{\partial r^{2}}\right)=\alpha_{\theta}^{-2}\left\|\frac{\partial X}{\partial r}\right\|^{2} .
\end{aligned}
$$

Referencing the array wrt its center, it comes:

$$
F_{12}=F_{21}=0 \text {. }
$$

Now using for instance the rough approximation $r_{1} / r_{k} \simeq 1-(k-1) L / r \cos \theta$ (in (43)) the following approximations of the norms of the derivative vectors are obtained:

$$
\begin{aligned}
& \left\|\frac{\partial X(r, \theta)}{\partial \theta}\right\|^{2} \cong \frac{L^{2}}{r^{2}} \sin ^{2} \theta \frac{\ell\left(\ell^{2}-1\right)}{12} \\
& \left\|\frac{\partial X(r, \theta)}{\partial r}\right\|^{2} \cong \frac{L^{4}}{r^{4}} \cos ^{2} \theta \sin ^{2} \theta \frac{\ell\left(\ell^{2}-1\right)}{12}
\end{aligned}
$$

leading finally to the following bound for $\operatorname{MSE}(\hat{r})$ :

$$
\operatorname{MSE}(\hat{r}) \cong \sigma_{\theta}^{2} \frac{12}{\ell\left(\ell^{2}-1\right)} \frac{r^{4}}{L^{2} \sin ^{2} \theta} .
$$

In (48), $\sigma_{\theta}^{2}$ is the variance of the estimated bearing for any subarray of the partitioned array. Note, that (48) extends (36). From this formula, it is obvious that using only a part of the (partitioned) array (e.g., the two extremities) for triangulation produces significant loss. It is also obvious from (36) and (48) that $\operatorname{MSE}(\hat{r})$ increases with $\ell$. Actually, the spherical wavefront is better approximated as $\ell$ increases but the source parameters are indepently ( $\Gamma$ diagonal) estimated on the subarrays. This effect is predominant. It is thus quite illusory to expect improved performance from partitioned processing in the ideal cases (fixed sources, coherent wavefront, etc.), even if the bound (48) may be pessimistic. They consist essentially in improved robustness and a reduced computation burden.

Actually, the true problem incomes with moving sources. Then the principle of the calculation of the MSE relies on the first order expansion of the gradient vector of the function $f$ :

$$
\nabla f(\hat{X}, \hat{\Omega}) \stackrel{1}{=} \nabla f(\hat{X}, \Omega)+H(\Omega, \hat{X}) \cdot(\hat{\Omega}-\Omega)
$$

with

$\mid \begin{array}{ll}\hat{\Omega} \quad: & \text { estimate of } \Omega \text { associated with } \hat{X} \\ \nabla f(\hat{X}, \hat{\Omega}): & \text { gradient vector of wrt } \Omega \text { calculated } \\ & \text { at the point } \hat{\Omega} \text { for } X=\hat{X} \\ H(\Omega, \hat{X}): & \text { Hessian matrix of } f \text { relative to } \Omega .\end{array}$

The elements of $\nabla f$ and $H$ are directly calculated from (39) and (41). Now, the following equality holds by definition of $(\hat{\Omega}, \hat{X})$ :

$$
\nabla f(\hat{X}, \hat{\Omega})=0
$$

therefore

$$
\hat{\Omega}-\Omega \simeq-H^{-1}(\Omega, X) \nabla f(\hat{X}, \Omega)
$$

so that finally

$$
\begin{aligned}
\operatorname{MSE}(\hat{\Omega})= & \mathrm{E}\left[(\hat{\Omega}-\Omega)(\hat{\Omega}-\Omega)^{\mathrm{t}}\right] \\
= & H^{-1}(\Omega, X) \mathrm{E}\left(\nabla f(\hat{X}, \Omega) \nabla^{t} f(\hat{X}, \Omega)\right) \\
& \times H^{-1}(\Omega, X) .
\end{aligned}
$$

Now, one has from (41):

$$
\nabla_{\Omega} f(\hat{X}, \Omega) \triangleq \nabla f(\hat{X}, \Omega)=\left\{\begin{array}{r}
2(X-\hat{X})^{t} \frac{\partial X}{\partial r_{1}} \\
2(X-\hat{X})^{t} \frac{\partial X}{\partial \cos \theta_{1}} \\
(X \triangleq \hat{X}(\Omega))
\end{array}\right.
$$

and, therefore

$$
\begin{aligned}
\mathbb{E}\left(\nabla f(\hat{X}, \Omega) \nabla^{t} f(\hat{X}, \Omega)\right) \\
=4\left(\begin{array}{c}
\frac{\partial X^{t}}{\partial r_{1}} \\
\frac{\partial X^{t}}{\partial \cos \theta_{1}}
\end{array}\right) \mathbb{E}\left[(X-\hat{X})(X-\hat{X})^{t}\right] \\
\quad \times\left(\frac{\partial X}{\partial r_{1}} \frac{\partial X}{\partial \cos \theta_{1}}\right)
\end{aligned}
$$

but

$$
\mathrm{E}\left[(X-\hat{X})(X-\hat{X})^{t}\right] \simeq L^{2} \cos ^{2} \theta \sigma_{\theta}^{2} I d_{\ell}
$$

yielding, finally,

$$
\begin{aligned}
\operatorname{MSE}(\hat{\Omega}) \simeq & 4 L^{2} \cos ^{2} \theta \sigma_{\theta}^{2} H^{-1}\left(\begin{array}{c}
\frac{\partial X^{t}}{\partial r_{1}} \\
\frac{\partial X^{t}}{\partial \cos \theta_{1}}
\end{array}\right) \\
& \times\left(\frac{\partial X}{\partial r_{1}} \frac{\partial X}{\partial \cos \theta_{1}}\right) H^{-1}
\end{aligned}
$$

The vectors $\partial X / \partial r_{1}$ and $\partial X / \partial \cos \theta_{1}$ are straightforwardly calculated (see Appendix C).

The study of the performance is illustrated by the three following tables. A moving source is considered with the following kinematic parameters: $v=10 \mathrm{~m} / \mathrm{s}$, $r_{0}=100 \mathrm{~km}, \theta_{0}=\pi / 4$, and $\beta=\pi / 4$. Then the values of the approximation of $\operatorname{var}(\hat{r})$ given by (51) are 
TABLE I

Statistical Performance of Range and Bearing Estimation With a Partitioned Array

\begin{tabular}{|c|c|c|}
\hline $\begin{array}{c}\rho= \\
N_{o p t}=10 \mathrm{~dB}\end{array}$ & calculated & estimated \\
\hline s.d. $(\hat{r})$ & $3321 \mathrm{~m}$ & $3333 \mathrm{~m}$ \\
\hline mean $(\hat{r})$ & & $100165 \mathrm{~m}$ \\
\hline s.d. $(\hat{\theta})$ & $1.7 \times 10^{-4} \mathrm{rad}$ & $1.7 \times 10^{-4} \mathrm{rad}$ \\
\hline
\end{tabular}

Note: $\ell=2, q=500$

TABLE II

Statistical Performance of Range and Bearing Estimation With a Partitioned Array

\begin{tabular}{|c|c|c|}
\hline $\begin{array}{l}\rho=0 \mathrm{~dB} \\
N_{\text {opt }}=3\end{array}$ & calculated & estimated \\
\hline s.d. $(\dot{r})$ & $1794 \mathrm{~m}$ & $10000 \mathrm{runs}$ \\
\hline mean $(\hat{r})$ & & $100060 \mathrm{~m}$ \\
\hline s.d. $(\dot{\theta})$ & $9.2 \times 10^{-5} \mathrm{rad}$ & $9 \times 10^{-5} \mathrm{rad}$ \\
\hline
\end{tabular}

Note: $\ell=2, q=500$

TABLE III

Statistical Performance of Range and Bearing Estimation With a Partitioned Array

\begin{tabular}{|c|c|c|}
\hline$\rho$ & $\rho=-10 \mathrm{~dB}$ & $\rho=0 \mathrm{~dB}$ \\
& $N_{o p t}=223$ & $N_{o p t}=95$ \\
\hline s.d. $(\hat{r})$ & $6896 \mathrm{~m}$ & $3267 \mathrm{~m}$ \\
\hline mean $(\hat{r})$ & $100727 \mathrm{~m}$ & $100228 \mathrm{~m}$ \\
\hline s.d. $(\hat{\theta})$ & $5.3 \times 10^{-4} \mathrm{rad}$ & $2.5 \times 10^{-4} \mathrm{rad}$ \\
\hline
\end{tabular}

Note: $\ell=20, q=50$.

compared with empirical estimation obtained by 10000 Monte-Carlo runs. The results are summarized in Tables I, II, and III.

These tables need some comments. The parameter $\Omega$ is estimated by maximizing the least-square functional defined by (49) and (51). This a nonlinear problem which is solved by using a standard MATLAB routine (Nelder-Meade algorithm).

The use of a partitioned array processing for range estimation instead of the whole array processing degrades slightly the performance in range estimation as seen on Tables I-III. However, a reduction of the computation burden and, overall, an enhanced robustness is expected.

Actually, the use of a partitioned array processing can seriously improve the robustness in range estimation for the following reasons.
1) The choice of the number of temporal integration (or snapshots) $N$ is much less critical.

2) The effects of physical factors like the spatial coherence of the wavefront, the array shape, etc. are generally less important for a short array.

3) The estimation of the CSM $\hat{R}$ for a large array suffers from major drawbacks (e.g., bias decoherence [14])

For all these reasons, the partitioned processing appears promising for large array. It is often referenced in the literature under the name "decentralized processing" (see e.g., [17-21]), but it represents only a very specific (and restrictive) approach to the general problem of distributed processings [22-24].

There are some variants of the partitioned processings. One of them is the Stansfield algorithm $[20,25]$ whose performance has been analyzed by M. Gavish and A. J. Weiss [26]. The interest of the Stansfield algorithm relies on the linearization of the $X$ vector [26]. It is then possible to use standard least-squares algorithms, i.e., with the notations of [26] consider the following (Stansfield) functional:

$$
F_{S T}(\hat{\Theta}, \mathbf{X})=\frac{1}{2}(A \mathbf{X}-\mathbf{b})^{\mathrm{t}} R^{-1}(A \mathbf{X}-\mathbf{b})
$$

where

$$
\begin{aligned}
& A \triangleq\left(\begin{array}{cc}
\sin \hat{\theta}_{1} & -\cos \hat{\theta}_{1} \\
\vdots & \vdots \\
\sin \hat{\theta}_{\ell} & -\cos \hat{\theta}_{\ell}
\end{array}\right) \\
& \mathbf{b} \triangleq\left(\begin{array}{l}
x_{S 1} \sin \hat{\theta}_{1}-y_{S 1} \cos \hat{\theta}_{1} \\
\vdots \\
x_{S \ell} \sin \hat{\theta}_{\ell}-y_{S \ell} \cos \hat{\theta}_{\ell}
\end{array}\right.
\end{aligned}
$$

$\left(X_{s i}, y_{s i}\right)$ are the Cartesian coodinates of the $i$ th sensor (here the center of the $i$ th subarray)

$$
R \triangleq \operatorname{diag}\left(r_{1}^{2}, \ldots, r_{\ell}^{2}\right)
$$

where $\mathbf{X}=\left(x_{t}, y_{t}\right)^{\mathrm{t}}$, the Cartesian coordinates vector.

The minimization of (48) with respect to $X$ leads to

$$
\hat{\mathbf{X}}_{S T}=\left(A^{\mathrm{t}} R^{-1} A\right)^{-1} A^{\mathrm{t}} R^{-1} \mathbf{b}
$$

provided that $R$ is known. Actually, even $R$ is not perfectly known, rough estimates of $R$ can be used.

The bias of $\hat{\Omega}$ (46) may be calculated in the same way, but it replaces the first-order expansion of the gradient $\nabla f(\hat{X}, \hat{\Omega})$ by a second-order one.

\section{DETECTION PERFORMANCE FOR MOVING SOURCES}

This section deals with the study of the detection performance of the classical (quadratic) $[1,27-30]$ 
receiver (21) in the case of a moving source. An exact calculation of the detection performance can be achieved at the expense of rather tedious calculations. They are presented in Appendix D and summarized below (with the notations of Appendix D):

$$
\begin{aligned}
P_{\mathrm{d}}= & \sum_{i=0}^{N / 2-1} A_{i}^{2}\left(\frac{N_{\mu}}{\alpha_{i}}+1\right) e^{-N_{\mu} / \alpha_{i}} \\
& +2 \sum_{i=0, i \neq j}^{N / 2-1} \sum_{j=0}^{N / 2-1} \frac{\alpha_{i} A_{i} A_{j}}{\alpha_{i}-\alpha_{j}} e^{-N_{\mu} / \alpha_{i}}
\end{aligned}
$$

with

$$
\mid \begin{aligned}
& \alpha_{i}=b p+\sigma\left|D_{i}^{*} D\right| \\
& A_{i}=\alpha_{i}^{N / 2-1} \prod_{p=0, p \neq i}^{N / 2-1}\left(\alpha_{i}-\alpha_{p}\right)^{-1} \\
& D \triangleq D_{\Omega_{M}} \quad(N \text { even }) .
\end{aligned}
$$

Since the calculation of $P_{\mathrm{fa}}$ is not affected by the source motion, it takes the classical form $[1,31,32]$ :

$$
P_{\mathrm{fa}}=e^{-N \mu / \beta} \sum_{t=0}^{N-1} \frac{1}{t !}\left(\frac{N \mu}{\beta}\right)^{\mathrm{t}}
$$

with $\beta=b p$.

It may be enlightening to consider the Gaussian approximation of the quadratic receiver even if it is a rather rough approximation. More precisely, the following approximation is considered (with the notations of Section III).

$$
\hat{P}\left(\Omega_{M}\right) \sim \mathcal{N}\left(m, \sigma^{2}\right)
$$

with

$$
\left\{\begin{array}{l}
m=\mathbb{E}\left(\hat{P}\left(\Omega_{M}\right)\right)=\frac{1}{N} \sum_{t=0}^{N-1} \operatorname{tr}\left(D_{\Omega_{M}}^{*} D_{\Omega_{M}}^{*} R_{t}\right) \\
\sigma^{2}=\operatorname{var}\left(\hat{P}\left(\Omega_{M}\right)\right)=\frac{1}{N^{2}} \sum_{t=0}^{N-1} \operatorname{tr}\left[\left(D_{\Omega_{M}} D_{\Omega_{M}}^{*} R_{t}\right)^{2}\right] .
\end{array}\right.
$$

With this approximation in mind, the probability of detection takes the following form:

$$
\begin{gathered}
P_{\mathrm{d}}=\operatorname{erfc}\left(\frac{\mu-m}{\sigma}\right), \\
\operatorname{erfc}(x) \triangleq \frac{1}{\sqrt{2 \pi}} \int_{x}^{\infty} e^{-u 2 / 2} d u .
\end{gathered}
$$

The probability of false alarm takes a similar form. Examinating (56) and (57) it is not hard to see that, for a given threshold $\mu$, the probability $P_{\mathrm{d}}$ increases at first (as $N$ increases) since the variance is decreasing and then decreases as the mean $m$ (and the variance) decreases and finally tends towards zero [7]. The optimal choice of $N$ would be the value of $N$ maximizing $P_{\mathrm{d}}$ but it is quite a simplistic approach since, on another hand, a larger $N$ allows us to choose a lower threshold $\mu$.

Therefore, the study of the detection performance for moving sources must be considered in terms

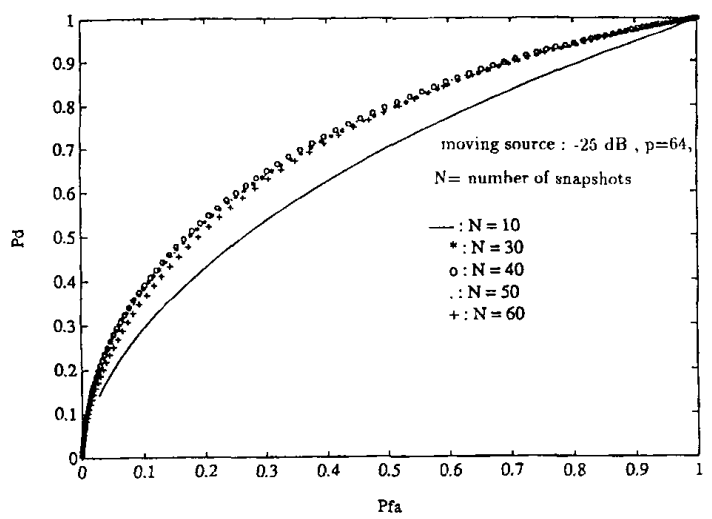

Fig. 15. Typical behavior of ROC curves for given source + array scenario and various values of $N\left(N_{1}<N_{\mathrm{opt}}<N_{2}\right)$.

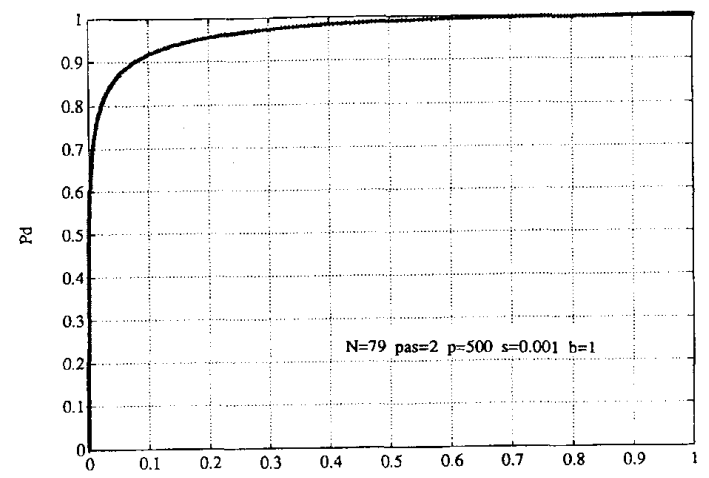

Fig. 16. ROC curve for a far moving source $\left(\theta_{0}=\pi / 4, \beta=\pi / 4\right.$, $\left.v=10 \mathrm{~m} / \mathrm{s}, r_{0}=10 \mathrm{~km}, \rho=-30 \mathrm{~dB}\right)$ and a large array $(p=500$, $d=\lambda / 2$ ).

of receiver operating characteristic (ROC) curves. Actually, for fixed source and array parameters, the ROC curves begin to increase with $N$ (i.e., $\operatorname{ROC}\left(N_{1}\right)>\operatorname{ROC}\left(N_{2}\right)$ for each couple $P_{\mathrm{d}}, P_{\mathrm{fa}}$ and $N_{1}>N_{2}$ ), attain a maximum value for a given value of $N$ (named $N_{\text {opt }}$ for the rest), and then decrease. This behavior is illustrated by Fig. 15. Obviously, the value of $N_{\text {opt }}$ depends upon the physical parameters of the source + array system, i.e., the kinematic parameters of the source, the number of sensors, and the signal-to-noise ratio.

In this spirit, the following result is rather surprising. For Figs. 16 and 17, the array is constitued of 500 equispaced sensors $(d=\lambda / 2)$, the source speed is $v=10 \mathrm{~m} / \mathrm{s}$ and its heading $\beta$ and its initial bearing are both equal to $\pi / 4$, its signal-to-noise ratio. $\rho$ is $-30 \mathrm{~dB}$.

For the first figure (Fig. 16) the initial source distance is $r_{0}=100 \mathrm{~km}$, while this distance is reduced to $r_{0}=10 \mathrm{~km}$ for the second experiment (Fig. 17). Then, for the long-range scenario (Fig. 16) the optimum value of $N$ is $N_{\mathrm{opt}}=79$, while for the close scenario (Fig. 17) it takes a very low value, i.e., $N=9$. This fact may be easily explained by considering the 


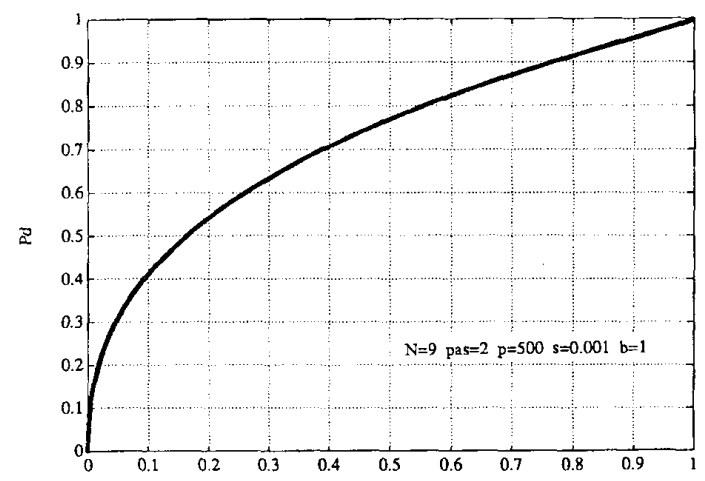

Fig. 17. ROC curve for a close moving source $\left(\theta_{0}=\pi / 4\right.$, $\left.\beta=\pi / 4, v=10 \mathrm{~m} / \mathrm{s}, r_{0}=10 \mathrm{~km}, \rho=-30 \mathrm{~dB}\right)$ and a large array $(p=500, d=\lambda / 2)$.

respective values of the elementary cells (in bearing and distance), i.e., for instance the values of the parameters $2 \theta_{3}$ and $2 r_{3}$ presented and calculated in Section II (see Fig. 18).

More generally, the source motions may seriously degrade the detection performance thus reducing drastically the interest of a direct processing of a very large array. Consider for instance the following problem. A far source is moving with the following kinematic parameters: $r_{0}=100 \mathrm{~km}, v=10 \mathrm{~m} / \mathrm{s}, \theta_{0}=$ $2 \pi / 3, \beta=\pi / 4$.

In a first time, a very large array is considered $(p=1000)$ as well as a very low signal-to-noise ratio $(\rho=-30 \mathrm{~dB})$. In this case, $N_{\mathrm{opt}}=43$ and the corresponding ROC curve is recorded.

In a second time, a much shorter array is considered since $p=50$. Then, a similar ROC curve is obtained for the following source and processing parameters: $\rho=0.0035$ and $N_{\mathrm{opt}}=731$. Therefore, the detection gain of the large array is approximately equal to $5 \mathrm{~dB}$ (exactly $5.44 \mathrm{~dB}$ ) while it should be equal to $13 \mathrm{~dB}$ (i.e., $\left.10 \log _{10}\left(\frac{1000}{50}\right),[1,30]\right)$. This value is rather low and is easily explained by the respective values of $N_{\text {opt }}$. The reduction of the array length is balanced by the increased value of $N_{\text {opt }}$. This conclusion is even reinforced by the source closeness. More philosophically, the array processing relies upon the spatio-temporal diversity [19] and, generally, these two diversities are antagonist. Therefore a compromise is necessary.

However, it is worth noting that this (classical) analysis is too simplistic. Actually, many informations from a large array may be used, especially for close sources (e.g., differential Doppler, Doppler rate [33, 34 , etc.). It seems that the direct processing of the whole array outputs can suffer from serious problems and this pleads for the use of partitioned processings.

This partitioned processings may include the use of dynamic informations relative to sources (Doppler, Doppler rate, etc.). But if a rather classical analysis of partitioned processings for source localization can provide a satisfying analysis of the performance for source localization, this is not true for the detection problem. Actually, the true detection problem does not correspond to the scholar study: a fixed source and a stationary array. The true problem we deal with is much more complex. In fact, the notion of point source must be replaced by the notion of source track. The analysis of the associated detection problems lead to complex and difficult problems involving data association, track-to-track associations, tracking and multiple hypotheses [35-38].

Therefore, the next section is simply considered as a brief introduction to vast and difficult problems.

\section{ON THE BEARING ASSOCIATION PERFORMANCE}

The general frame of this section is completely similar to Section IVB. The array has been divided
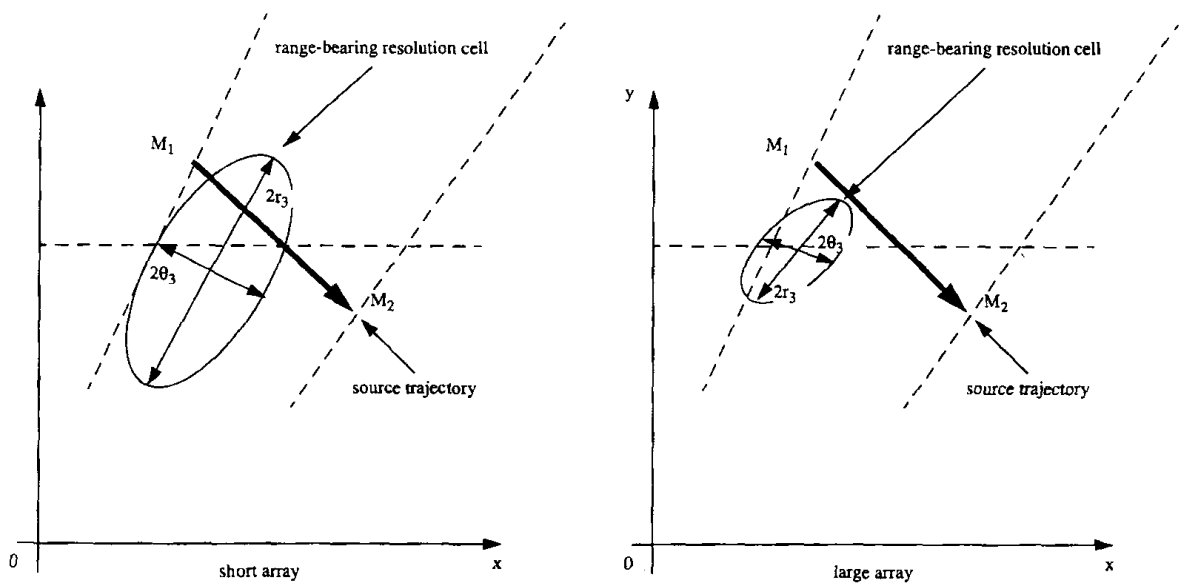

Fig. 18. Comparison of range-bearing resolution cells for short and large array (array center at origin 0 ). 


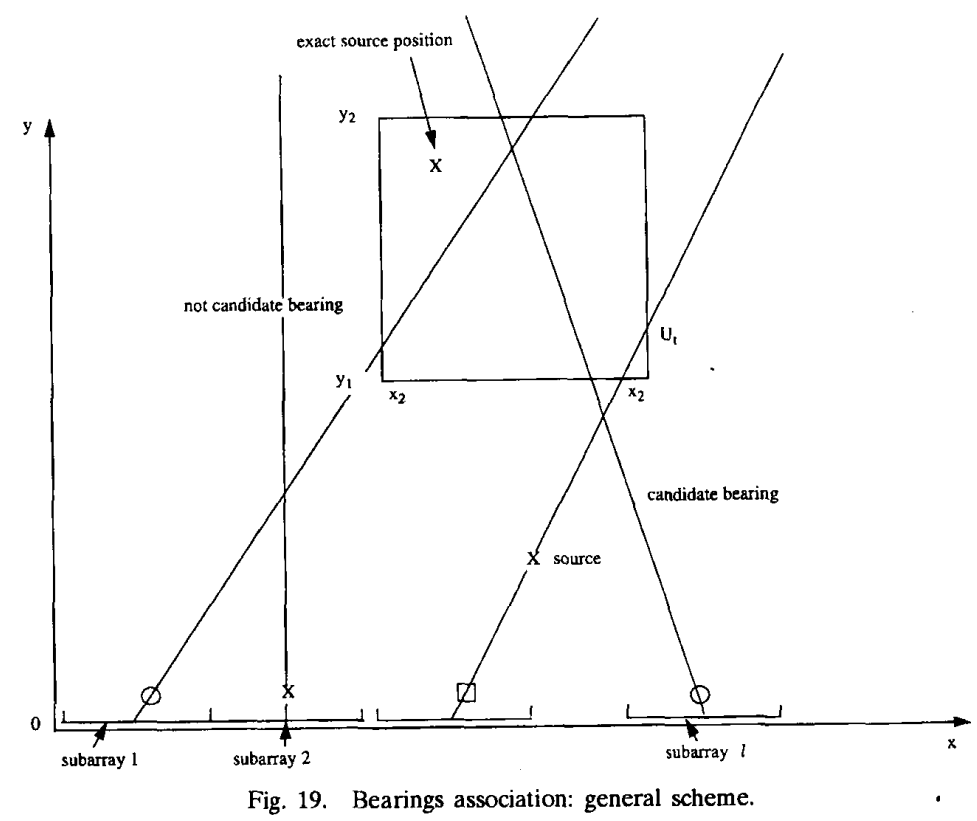

into $\ell$ subarrays. As previously, the plane wave assumption is made on each subarray and, for this section, the observation is constituted of a sequence of $\ell$ estimated bearings $\left\{\hat{\theta}_{1}, \hat{\theta}_{2}, \ldots, \hat{\theta}_{\ell}\right\}$.

The general association problem stands as follows. $P:$ is it possible to fit the observation sequence $\left\{\hat{\theta}_{1}, \hat{\theta}_{2}, \ldots, \hat{\theta}_{\ell}\right\}$ to a model $\left(\theta_{1}, \theta_{2}, \ldots, \theta_{\ell}\right)$ with

$$
\theta_{2}=f_{2}\left(\theta_{1}, r_{1}\right), \ldots, \theta_{\ell}=f_{\ell}\left(\theta_{1}, r_{1}\right)
$$

(the functions $f_{2}, \ldots, f_{\ell}$ being defined by (38)).

Let $\hat{\Theta}$ and $\Theta$, the observation and model vectors be defined by

$$
\begin{aligned}
& \hat{\Theta} \triangleq\left(\hat{\theta}_{1}, \hat{\theta}_{2}, \ldots, \hat{\theta}_{\ell}\right)^{\mathrm{t}} \\
& \Theta \triangleq\left(\theta_{1}, \theta_{2}, \ldots, \theta_{\ell}\right)^{\mathrm{t}}
\end{aligned}
$$

and define the association procedure as below [17]:

$$
\|\hat{\Theta}-\Theta\|^{2} \underset{H_{0}}{\lessgtr} d
$$

In (58), the scalar $d$ is the association threshold.

The association performance may be easily calculated under the Gaussian hypotheses. Let us consider, on a first time, the following likelihood ratio:

$$
\frac{P\left(\hat{\Theta} \mid H_{1}\right)}{P\left(\hat{\Theta} \mid H_{0}\right)} \lessgtr_{H_{0}}^{H_{1}} \mu
$$

with

$$
\begin{aligned}
& P\left(\hat{\Theta} \mid H_{1}\right)=\frac{1}{\sqrt{2 \pi} \sigma_{1}^{P}} \exp \left(-\frac{1}{2 \sigma_{1}^{p}}\|\hat{\Theta}-\Theta\|^{2}\right) \\
& P\left(\hat{\Theta} \mid H_{0}\right)=\frac{1}{\sqrt{2 \pi} \sigma_{0}^{p}} \exp \left(-\frac{1}{2 \sigma_{0}^{p}}\|\hat{\Theta}-\Theta\|^{2}\right)
\end{aligned}
$$

leading to the following association test:

$$
\|\hat{\Theta}-\Theta\|^{2} \underset{H_{0}}{\stackrel{H_{1}}{\lessgtr}}-2\left(\frac{1}{\sigma_{1}^{p}}-\frac{1}{\sigma_{0}^{p}}\right)^{-1} \log \left(\mu\left(\frac{\sigma_{1}}{\sigma_{0}}\right)^{p}\right)=\mu^{\prime} .
$$

Therefore under the Gaussian hypotheses (for both $H_{1}$ and $H_{0}$ ) the likelihood ratio simply results in (59). The association performance are then directly deduced, i.e.,

with

$$
P_{\mathrm{d}}=\int_{0}^{\mu^{\prime}} P_{\chi^{2}, \sigma_{1}^{2}}(x) d x
$$

$$
\left\{\begin{array}{l}
P \chi^{2}, \sigma^{2}(x)=\frac{1}{2^{\ell / 2} \Gamma(\ell / 2)} x^{(\ell-2) / 2} e^{-x / 2 \sigma^{2}} Y(x) \\
Y(x)=1 \text { if } x \in \mathbb{R}^{+}, 0 \text { else }
\end{array}\right.
$$

$\Gamma$ : gamma function

(i.e., $\left.\Gamma(n / 2)=\int_{0}^{\infty} y^{(n-2) / 2} e^{-y} d y\right)$

and similarly

$$
P_{\mathrm{fa}}=\int_{0}^{\mu^{\prime}} P \chi^{2}, \sigma_{0}^{2}(x) d x .
$$

Obviously, the probability of association $P_{a d}(59)$ is directly related with the value of the threshold $d$ (the validation gate). The calculation of the ROC curves for the association test is straightforwardly deduced from (56) and it is easily seen that the association performance is simply depending on the ratio $\sigma_{1} / \sigma_{0}$. Actually, this analysis is quite (and too) simplistic. So, this problem must be analyzed by using the general frame of probalistic data association (PDA for the sequel) as developed in $[36,39,40]$. More precisely, the following structure is considered (and depicted in Fig. 19). 


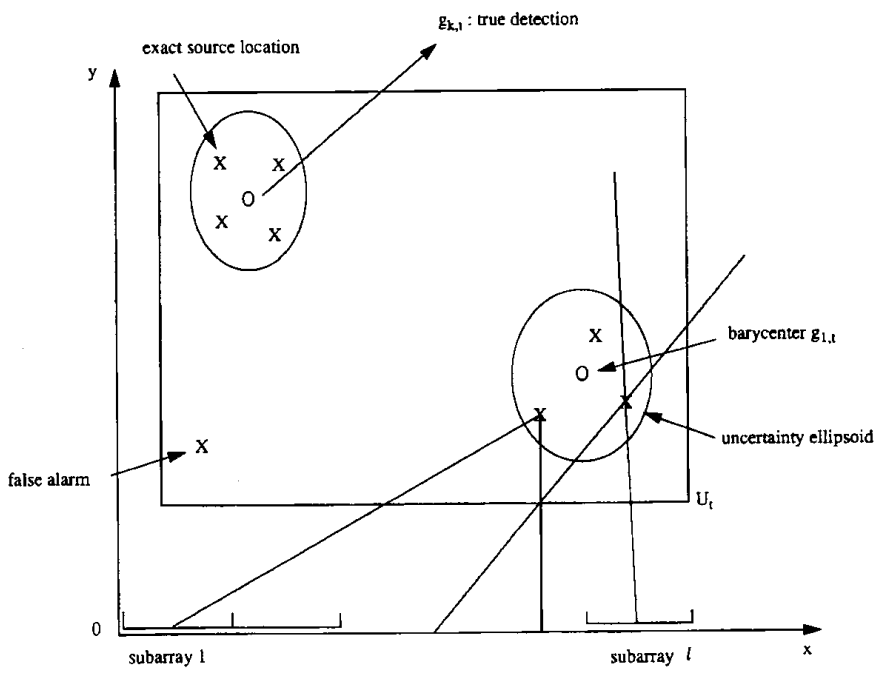

Fig. 20. Definition of true detection and false alarms.

1) The measures and the models are indexed by the time (current index $t$ ).

2) $U_{t}$ is a domain of $R^{2}$ e.g., $\left[x_{1}, x_{2}\right] \times\left[y_{1}, y_{2}\right]$. The choice of the bounds of the domain results from performance analysis, operations analysis, etc.

3) The array is partitioned. There is a first elementary detection step for each subarray.

4) $m_{t}$ estimated measures are candidate, an elementary measure is an (uncertainty) ellipsoid containing at least one intersection of lines issued from the subarrays. It is worth centering the ellipsoid on the barycenters of the line intersections (Fig. 20).

5) Among the $m_{t}$ measures falling in $U_{t}, m_{t}^{d}$ and $m_{t}^{f}$ correspond, respectively, to true detections and false alarms.

Based on the ideas of C. Jauffret [40,39], the following statistical model of the observations is considered.

1) $A_{1}$ : there is at most one true detection on the domain $U_{t}$ (however this hypothesis may be easily extended),

2) $A_{2}$ : conditionally to a true detection in the cell $j\left(H_{j, t}\right)$ the density of a barycenter $\mathbf{g}_{j, t}$ is Gaussian, i.e.,

$$
\begin{aligned}
p\left(\mathbf{g}_{j, t} \mid H_{j, t}\right)= & \frac{1}{P_{U_{t}}}(2 \pi)^{-d / 2}(\operatorname{det} \Sigma)^{-1 / 2} \\
& \times \exp \left\{-\frac{1}{2}\left\|\mathbf{g}_{j, t}-h_{j, t}(\mathbf{X})\right\|_{\Sigma^{-1}}^{2}\right\}
\end{aligned}
$$

where in the simpler case $d=2, \mathbf{X}$ is a state vector characterizing the source trajectory, $\Sigma$ is the uncertainty ellipsoid, and $H_{j, t}$ represents the hypothesis: $g_{j, t}$ is a true detection (i.e., the exact source location is situated in ellipsoid associated with $g_{j}$ ).
Finally:

$$
\begin{aligned}
P_{U_{t}} \triangleq & \int_{U_{t}}(2 \pi)^{-d / 2}(\operatorname{det} \Sigma)^{-1 / 2} \\
& \quad \times \exp \left\{-\frac{1}{2}\left\|\mathbf{g}-h_{j, t}(\mathbf{X})\right\|_{\Sigma^{-1}}^{2}\right\} d \mathbf{g} .
\end{aligned}
$$

3) $A_{3}$ : the false alarms are uniformly distributed in $U_{t}$, i.e.,

$$
p\left(\mathbf{g}_{j, t} \mid H_{i, t}\right)=\frac{1}{u} \quad(j \neq i)
$$

and the number of false alarms is Poisson distributed, i.e.,

$$
P\left(m_{t}^{f}=k\right) \triangleq(\lambda u)^{k} \frac{e^{-\lambda u}}{k !} \triangleq \mu_{u}(k)
$$

(the parameter $\lambda$ is fixed by statistical considerations).

Then, if the observation at time $t$ is constituted of $m_{t}$ barycenters $\mathbf{g}_{1, t}, \ldots, \mathbf{g}_{m_{t}, t}$ (represented by the vector $\mathbf{G}_{t}$ ), a general expression of the likelihood stands as follows:

$$
\begin{aligned}
p\left(\mathbf{G}_{t} \mid \mathbf{X}\right)= & u^{-m_{t}}\left(1-P_{\mathrm{d}}\right) \mu_{u}\left(m_{t}\right)_{m_{t}} \\
& +u^{1-m_{t}} \frac{P_{\mathrm{d}}}{P_{u_{t}}} \frac{\mu_{u}\left(m_{t}-1\right)}{m_{t}} \sum_{j=1}^{m_{t}}(2 \pi)^{-d / 2}(\operatorname{det} \Sigma)^{-1 / 2} \\
& \times \exp \left\{-\frac{1}{2}\left\|\mathbf{g}_{j, t}-h_{j, t}(\mathbf{X})\right\|^{2}\right\}
\end{aligned}
$$

For the sake of completness, an elementary proof (based on [40]) is presented in Appendix E.

This data association approach may be directly extended to dynamic data association. More precisely, consider a source in rectilinear and uniform motion, then its trajectory is characterized by a state vector $\mathbf{X}$ [41] (e.g., initial location and velocity vector). Assuming the various observations $\mathbf{G}_{\boldsymbol{t}}$ statistically independent 
(the collection of these vectors is denoted $\mathbf{G}$ ), then the likelihood takes the following form:

$$
p(\mathbf{G} \mid \mathbf{X})=\prod_{t} p\left(\mathbf{G}_{t} \mid \mathbf{X}\right) .
$$

It is then possible to estimate the vector $\mathbf{X}$ maximizing the functional $p(\mathbf{G} \mid \mathbf{X})$ (in (60)) by using a numerical (gradient's like or Newton) algorithm. The generalized likelihood ratio takes then the following form [40]:

$$
\Lambda(\mathbf{G} \mid \hat{\mathbf{X}}) \triangleq \frac{p\left(\mathbf{G} \mid \hat{\mathbf{X}}, H_{1}\right)}{p\left(\mathbf{G} \mid H_{0}\right)}
$$

where $\hat{\mathbf{X}}$ denotes the $\mathbf{X}$ vector maximizing the likelihood function (64).

This approach may be extended to multiple sources (by increasing the dimension of $\mathbf{X}$ ). Another view of the problem is track-to-track association [37, 38].

\section{CONCLUSION}

The performance of wavefront curvature methods for range estimation has been investigated along with associated detection problems. The main originality of this paper relies on the consideration of the source motions. However, this simple (and quite natural) hypothesis has important practical consequences. In particular, the benefits of a direct processing of the whole array of sensors (detection gain, variance of the range estimates) are seriously mitigated by the lack of robustness for moving sources, which is the general case. Furthermore, the performance analysis presented in this paper (for large array) is certainly quite optimistic since it does not take into account physical perturbations like wavefront coherence, array calibration, bias decoherence, etc.

For all these reasons, a direct processing is quite questionable and can be advantageously replaced by a partitioned array processing. The performances of such partitioned processing have been studied and compared with the direct processing one. It seems, therefore, that it represents a promising way for the processing of large arrays.

\section{APPENDIX A}

The matrix $\partial g / \partial \Omega$ presents the general following form (8) and (9):

$$
\frac{\partial g}{\partial \Omega}=\left(\begin{array}{ll}
G & S \\
S & \varepsilon
\end{array}\right)
$$

therefore

$$
\left(\frac{\partial g}{\partial \Omega}\right)^{-1}=\frac{1}{G \varepsilon-S^{2}}\left(\begin{array}{rr}
\varepsilon & -S \\
-S & G
\end{array}\right) .
$$

Now numeral considerations assert that $S^{2}$ is little in regard to $G \varepsilon$, therefore

$$
\left(\frac{\partial g}{\partial \Omega}\right)^{-1} \sim\left(\begin{array}{cc}
G^{-1} & \frac{-S}{G \varepsilon} \\
\frac{-S}{G \varepsilon} & \varepsilon^{-1}
\end{array}\right)
$$

providing thus the following approximation:

$$
\begin{aligned}
\hat{r}_{M}-r_{M} & \simeq \frac{1}{\varepsilon}(1-S / G) \frac{\partial P}{\partial r} \\
& \simeq\left(\frac{\partial g}{\partial r}\right)_{r=r_{M}}^{-1} g\left(r_{M}, \hat{R}\right)
\end{aligned}
$$

so that finally (assuming the bias null):

$$
\operatorname{var}\left(\hat{r}_{M}\right) \simeq\left(\frac{\partial g}{\partial r / r=r_{M}}\right)^{-2} \mathbb{E}\left[g^{2}\left(r_{M}, \hat{R}\right)\right]
$$

Now, the following equalities hold from (21)-(26)

$$
\begin{gathered}
g\left(r_{M}, \hat{R}\right)=2 \operatorname{Re}\left(U_{r_{M}}^{*} \hat{R} D_{\Omega_{M}}\right) \\
\frac{\partial g}{\partial r} \mid r=r_{M}=2\left[\operatorname{Re}\left(V_{r_{M}}^{*} R D_{\Omega_{M}}\right)+U_{r_{M}}^{*} R U_{r_{M}}\right] .
\end{gathered}
$$

Furthermore, classical calculations [7] provide:

$$
\begin{aligned}
& \mathbb{E}\left(\left[\operatorname{Re}\left(U_{r_{M}}^{*} \hat{R} D_{\Omega_{M}}\right)\right]^{2}\right) \\
& =\frac{1}{N^{2}}\left[\sum_{t=0}^{N-1} \operatorname{tr}\left(\left(A R_{t}\right)^{2}\right)+\left[\sum_{t=0}^{N-1} \operatorname{tr}\left(A R_{t}\right)\right]^{2}\right] .
\end{aligned}
$$

The above equality relies on the following classical formula:

$$
\begin{aligned}
& \mathbb{E}\left[\left(X^{*} A X\right)\left(X^{*} B X\right)\right] \\
& =\operatorname{tr}(B R A R)+\operatorname{tr}(A R) \operatorname{tr}(B R) \\
& R \triangleq \operatorname{cov}(X) ; \quad X: \mathcal{N}(0, R)
\end{aligned}
$$

itself resulting from the expression of the characteristic function $\Phi$ of the quadratic form $X^{*} A X$, i.e.,

$$
\Phi(u)=(\operatorname{det}(I d-i u A R))^{-1} .
$$

As is seen later, this formula is instrumental for the calculation of the detection performance (see Appendix D).

Using (65), the expectation of $g^{2}\left(r_{M}, \hat{R}\right)$ may be easily calculated, yielding:

$$
\mathbb{E}\left(g^{2}\left(r_{M}, \hat{R}\right)\right)=\frac{1}{N^{2}} \sum_{m=0}^{N-1} \mathbb{E}\left[X_{n}^{*} A X_{n} X_{m}^{*} A X_{m}\right]
$$


with

$$
\text { (with: } \begin{aligned}
A= & \left.D_{\Omega_{M}} U_{r_{M}}^{*}+U_{r_{M}} D_{\Omega_{M}}^{*}\right) \\
= & \frac{1}{N^{2}} \sum_{t=0}^{N-1}\left\{\operatorname{tr}\left[\left(A R_{t}\right)^{2}\right]+\operatorname{tr}^{2}\left(A R_{t}\right)\right\} \\
& +\frac{1}{N^{2}} \sum_{t=0}^{N-1} \sum_{t^{\prime}=0, t^{\prime} \neq t}^{N-1} \operatorname{tr}\left(A R_{t}\right) \operatorname{tr}\left(A R_{t^{\prime}}\right) \\
= & \frac{1}{N^{2}} \sum_{t=0}^{N-1} \operatorname{tr}\left[\left(A R_{t}\right)^{2}\right]+\frac{1}{N^{2}}\left(\sum_{t=0}^{N-1} \operatorname{tr}\left(A R_{t}\right)\right)^{2} .
\end{aligned}
$$

The expression $(28)$ of $\operatorname{var}\left(\hat{r}_{M}\right)$ results directly from (27), (66), (70).

\section{APPENDIX B}

A simple proof of the formula (36) is now presented. Since the range estimation $\hat{r}_{2}$ given by (35) is a differentiable function of the observation vector $\left(\hat{\theta}_{1}, \hat{\theta}_{2}\right)^{\mathbf{t}}$, in order to calculate the term $\mathbb{E}\left(\hat{r}_{2}-r_{2}\right)^{2} \triangleq \operatorname{MSE}\left(\hat{r}_{2}\right)$ a first-order expansion of $\hat{r}_{2}$ is considered.

For this purpose, consider the first order expansion of the observation vector:

$$
\mid \begin{aligned}
& \hat{\theta}_{1}=\theta_{1}+\delta_{1} \\
& \hat{\theta}_{2}=\theta_{2}+\delta_{2} \quad\left(\delta_{1}=\delta_{\theta_{1}}, \delta_{2}=\delta_{\theta_{2}}\right)
\end{aligned}
$$

then, it comes:

$$
\begin{gathered}
\sin \left(\hat{\theta}_{1}\right) \stackrel{1}{=} \sin \theta_{1}+\delta_{1} \cos \theta_{1} \\
\sin \left(\hat{\theta}_{2}-\hat{\theta}_{1}\right) \triangleq \sin \left(\theta_{2}-\theta_{1}\right)\left[1+\left(\delta_{2}-\delta_{1}\right) \tan ^{-1}\left(\theta_{2}-\theta_{1}\right)\right]
\end{gathered}
$$

so that

$$
\begin{aligned}
\hat{r}_{2} \stackrel{1}{=} & \frac{L}{\sin \left(\theta_{2}-\theta_{1}\right)} \\
& \times\left[\sin \theta_{1}+\delta_{1} \cos \theta_{1}-\left(\delta_{2}-\delta_{1}\right) \frac{\sin \theta_{1}}{\tan \left(\theta_{2}-\theta_{1}\right)}\right] .
\end{aligned}
$$

Denoting $\delta r_{2}$ the range error $\left(\delta r_{2} \triangleq \hat{r}_{2}-r_{2}\right)$, direct calculations yield:

$$
\delta r_{2}=\frac{L}{\sin \left(\theta_{1}-\theta_{2}\right)}\left[\delta_{1} \cos \theta_{1}-\left(\delta_{2}-\delta_{1}\right) \frac{\sin \theta_{1}}{\tan \left(\theta_{2}-\theta_{1}\right)}\right]
$$

and, thus

$$
\frac{\delta r_{2}}{r_{2}}=\delta_{1} \frac{\cos \theta_{1}}{\sin \theta_{1}}-\left(\delta_{2}-\delta_{1}\right) \frac{1}{\tan \left(\theta_{2}-\theta_{1}\right)}
$$

or, equivalently,

$$
\frac{\delta r_{2}}{r_{2}}=\left(\frac{1}{\tan \theta_{1}}-\frac{1}{\tan \left(\theta_{1}-\theta_{2}\right)}\right) \delta_{1}+\frac{1}{\tan \left(\theta_{1}-\theta_{2}\right)} \delta_{2} .
$$

Assuming the estimation noises $\delta_{1}$ and $\delta_{2}$ uncorrelated and of the same variances, a first-order approximation of the MSE is directly deduced:

$$
\begin{aligned}
\operatorname{MSE}\left(\hat{r}_{2}\right) \stackrel{1}{=} r_{2}^{2}[ & \left(\frac{1}{\tan \theta_{1}}-\frac{1}{\tan \left(\theta_{1}-\theta_{2}\right)}\right)^{2} \\
& \left.+\frac{1}{\tan \left(\theta_{1}-\theta_{2}\right)}\right] \sigma_{\theta}^{2} .
\end{aligned}
$$

A further step of approximation is the long distance hypothesis $[15,16]$ (i.e., $\tan \theta_{1} \gg \tan \left(\theta_{1}-\theta_{2}\right)$ ) which results in

$$
\operatorname{MSE}\left(\hat{r}_{2}\right) \simeq \frac{2 r^{2} \sigma_{\theta}^{2}}{\tan ^{2}\left(\theta_{1}-\theta_{2}\right)}
$$

Another approximation is usually made:

$$
\sin \left(\theta_{2}-\theta_{1}\right)=\frac{L}{r_{2}} \sin \theta_{1} \simeq \frac{L}{r} \sin \theta
$$

(for far sources $r_{1} \simeq r_{2}=r, \theta_{1} \simeq \theta_{2}=\theta$ ) and

$$
\begin{aligned}
\cos \left(\theta_{2}-\theta_{1}\right) & =\left(1-\sin ^{2}\left(\theta_{2}-\theta_{1}\right)\right)^{1 / 2} \\
& \simeq 1-\frac{1}{2} \frac{L^{2}}{r^{2}} \sin ^{2} \theta
\end{aligned}
$$

yielding the following classical $[15,16]$ approximation:

$$
\operatorname{MSE}\left(\hat{r}_{2}\right) \simeq 2 \frac{r^{4}}{L^{2}} \frac{1}{\sin ^{-2} \theta} \sigma_{\theta}^{2} .
$$

An approximation of the bias is obtained in the same way but a second-order expansion must, this time, be considered:

$$
\sin \hat{\theta}_{1} \stackrel{2}{=} \sin \theta_{1}+\delta_{1} \cos \theta_{1}-\frac{\delta_{1}^{2}}{2} \sin \theta_{1}+0\left(\delta_{1}^{2}\right)
$$

which yields

$$
L \sin \hat{\theta}_{1} \stackrel{2}{=} L \sin \theta_{1}\left(1+\delta_{1} \frac{1}{\tan \theta_{1}}-\frac{\delta_{1}^{2}}{2}\right)
$$

and similarly:

$$
\begin{aligned}
\sin \left(\theta_{1}-\hat{\theta}_{2}\right) \stackrel{2}{=} \sin \left(\theta_{1}-\theta_{2}\right)[ & 1+\left(\delta_{1}-\delta_{2}\right) \frac{1}{\tan \left(\theta_{2}-\theta_{1}\right)} \\
& \left.-\frac{\left(\delta_{1}-\delta_{2}\right)^{2}}{2}\right]
\end{aligned}
$$

so, that:

$$
\mathrm{E}\left(\hat{r}_{2}\right) \simeq r_{2}\left(1+2 \sigma_{\theta}^{2} \frac{1}{\tan ^{2}\left(\theta_{1}-\theta_{2}\right)}\right)
$$

$\left(\tan \left(\theta_{1}-\theta_{2}\right) \ll \tan \theta_{1}, \delta_{1}\right.$ and $\delta_{2}$ uncorrelated). Finally, the following approximation of the bias is obtained

$$
\operatorname{bias}\left(\hat{r}_{2}\right) \simeq 2 r_{2} \sigma_{\theta}^{2} \frac{1}{\tan ^{2}\left(\theta_{1}-\theta_{2}\right)}
$$


but: $\tan \left(\theta_{1}-\theta_{2}\right) \simeq L \sin \theta / r$, and, consequently:

$$
\mathbb{E}\left(\hat{r}_{1}\right) \simeq 2 \sigma_{\theta}^{2} \frac{r^{3}}{L^{2} \sin ^{2} \theta} .
$$

APPENDIX C

$$
X \triangleq\left(\sin \theta_{2}, \ldots, \sin \theta_{\ell}\right)^{t}
$$

then

$$
x_{k} \triangleq L \sin \left(\theta_{k}\right)
$$

Direct calculations yield:

$$
\begin{aligned}
\frac{\partial x_{k}}{\partial r_{1}}= & x_{k} \cdot\left(\frac{1}{r_{1}}-\frac{r_{1}+(k-1) L \cos \theta_{1}}{r_{k}^{2}}\right) \\
\frac{\partial x_{k}}{\partial \cos \theta_{1}}= & -x_{k} \cdot\left(\frac{\cos \theta_{1}}{\sin ^{2} \theta_{1}}+\frac{(k-1) L r_{1}}{r_{k}^{2}}\right) \\
\frac{\partial^{2} x_{k}}{\partial r_{1}^{2}}= & \frac{1}{x_{k}} \cdot\left(\frac{\partial x_{k}}{\partial r_{1}}\right)^{2} \\
& +x_{k}\left(-\frac{1}{r_{1}^{2}}-\frac{1}{r_{k}^{2}}+\frac{2\left(r_{1}+(k-1) L \cos \theta_{1}\right)^{2}}{r_{k}^{4}}\right) \\
\frac{\partial^{2} x_{k}}{\partial \cos ^{2} \theta_{1}}= & \frac{1}{x_{k}} \cdot\left(\frac{\partial x_{k}}{\partial \cos \theta_{1}}\right)^{2} \\
& +x_{k}\left(-\frac{\left(1+\cos ^{2} \theta_{1}\right)}{\sin ^{4} \theta_{1}}+\frac{2\left((k-1) L r_{1}\right)^{2}}{r_{k}^{4}}\right) \\
\frac{\partial^{2} x_{k}}{\partial r_{1} \partial \cos \theta_{1}}= & \frac{1}{x_{k}}\left(\frac{\partial x_{k}}{\partial r_{1}} \times \frac{\partial x_{k}}{\partial \cos _{1}}\right) \\
& -(k-1) L x_{k}\left[\frac{1}{r_{k}^{2}}+\frac{2 r_{1}\left(r_{1}+(k-1) L \cos \theta_{1}\right)}{r_{k}^{4}}\right]
\end{aligned}
$$

\section{APPENDIX D}

This Appendix is devoted to the calculation of $P_{\mathrm{d}}$ and $P_{\mathrm{fa}}$ in the unique source case $\left[7,7^{\prime}\right]$. The quadratic receiver (21) takes the following form for a moving source (21)-(23):

$$
\hat{P}\left(\theta_{M}, r_{M}\right)=\frac{1}{N} \sum_{t=0}^{N-1} X_{t}^{*} D D^{*} X_{t}
$$

with

$$
D \triangleq D_{\theta_{M}, r_{M}}
$$

We are mainly concerned with the calculation of the probability density of $\hat{P}$ under $H_{1}$ (source present). The calculation of the characteristic function constitutes the basic tool.

Let $\Phi_{1, t}(u)$ be the characteristic function of $\Delta_{t}\left(\Delta_{t} \triangleq X_{t}^{*} D D^{*} X_{t}\right)$ under $H_{1}$, then

$$
\begin{aligned}
\Phi_{1, t}(u) & \triangleq \mathbb{E}\left(\exp \left(i u \Delta_{t}\right) \mid H_{1}\right) \\
& =\left(\operatorname{det}\left(I d-i u D D^{*} R_{t}\right)\right)^{-1}, \quad i^{2}=1
\end{aligned}
$$

with

$$
R_{t}=\sigma D_{t} D_{t}^{*}+b I d \quad\left(D_{t} \triangleq D_{\theta_{t}, r_{t}}\right)
$$

so, that

$$
\mathbf{\Phi}_{1, t}(u)=\left(\operatorname{det}\left[I d-i u b D D^{*}-i u \sigma\left|D^{*} D_{t}\right|^{2}\right]\right)^{-1} .
$$

Now, the following first-order (exact) expansion of $\operatorname{det}(A+\lambda B)$ holds (rank $B=1$ ):

$$
\operatorname{det}(A+\lambda B)=\operatorname{det} A+\lambda \operatorname{det} A \operatorname{tr}\left(A^{-1} B\right) .
$$

Thus, denoting:

$$
\mid \begin{aligned}
& A=I d-i u b D D^{*} \\
& B=D D_{t}^{*} \\
& \lambda=-i u \sigma
\end{aligned}
$$

it comes:

$$
\begin{aligned}
& \operatorname{det} A=1-i u b p \\
& A^{-1}=I d+\alpha D D^{*} \\
& \alpha=i u b(1-i u b p)^{-1}
\end{aligned}
$$

yielding finally:

$$
\boldsymbol{\Phi}_{1, t}^{-1}(u)=1-i u\left(b p+\sigma\left|D_{t}^{*} D\right|^{2}\right)
$$

The characteristic function $\Phi_{1}$ of $N \hat{P}\left(\theta_{M}, r_{M}\right)$ is then directly deduced, i.e.,

$$
\Phi_{1}(u)=\prod_{t=0}^{N-1} \Phi_{1, t}(u)=\prod_{t=0}^{N-1} \frac{1}{1-i \alpha_{t} u}
$$

with

$$
\alpha_{t}=b p+\sigma\left|D_{t}^{*} D\right|^{2} .
$$

Thanks to the source motion model, the following equality trivially holds

$$
\alpha_{t}=\alpha(N-1-t) .
$$

Therefore, two cases must be considered for the calculation of $P_{\mathrm{d}}$.

1) $N$ is even, then:

$$
\Phi_{1}(u)=\left(\prod_{t=0}^{N / 2-1}\left(1-i \alpha_{t} u\right)^{-1}\right)^{2}
$$

2) $N$ is odd, then:

$$
\mathbf{\Phi}_{1}(u)=\frac{1}{1-i\left(b_{p}+\sigma p^{2}\right) u}\left(\prod_{t=0}^{(N-1) / 2-1}\left(1-i \alpha_{t} u\right)^{-1}\right)^{2} \text {. }
$$

Consider now, for instance, the case $N$ even. Let $\Psi_{1}$ the auxiliary function defined by

$$
\boldsymbol{\Phi}_{1}(u)=\mathbf{\Psi}_{1}^{2}(u)
$$


then

$$
\mathbf{\Psi}_{1}(u)=\prod_{t=0}^{N / 2-1}(1-i \alpha u)^{-1}=\sum_{t=0}^{N / 2-1} \frac{A_{t}}{1-i \alpha_{t} u}
$$

with

$$
A_{t}=\left(\alpha_{t}\right)^{N / 2-1} \prod_{j=0, j \neq t}^{N / 2-1}\left(\alpha_{t}-\alpha_{j}\right)^{-1}
$$

Now, the function $\Psi_{1}(u)$ is the Fourier transform of the function $P_{1}(x)$, i.e.,

$$
\Psi_{1}(u) \stackrel{\mathrm{TF}}{\rightleftharpoons} P_{1}(x)=\sum_{t=0}^{N / 2-1} Y(x) \frac{A_{t}}{\alpha_{t}} e^{-x / \alpha_{t}}
$$

where $Y(x)$ is the Heaviside function.

This simple remark constitutes the trick of the calculation of the probability of detection $P_{\mathrm{d}}$. Actually one has now

$$
\mathbf{\Phi}_{1}(u) \stackrel{\mathrm{TT}}{\rightleftharpoons} P_{1}^{(2)}(x) \triangleq P_{1}(x) \otimes P_{1}(x)
$$

(the symbol $\otimes$ means convolution) so, that:

$$
P_{1}^{(2)}(x)=\sum_{i=0}^{N / 2-1} \sum_{j=0}^{N / 2-1} \frac{A_{i} A_{j}}{\alpha_{i} \alpha_{j}} \int_{0}^{x} e^{-y / \alpha_{i}} e^{-(x-y) / \alpha_{j}} d y .
$$

The calculation of the integral in the above formula is direct yielding

$$
\begin{aligned}
P_{1}^{(2)}(x)= & \sum_{i=0}^{N / 2-1} \frac{A_{i}^{2}}{\alpha_{i}^{2}} Y(x) x e^{-x / \alpha_{i}} \\
& +2 \sum_{i=0 ; i \neq j}^{N / 2-1} \frac{A_{i} A_{j}}{\alpha_{i}-\alpha_{j}} Y(x)
\end{aligned}
$$

Now, one has

$$
P_{\hat{p} / H_{1}}(x)=N P_{1}^{(2)}(N x),
$$

so, using the previous results, $P_{\mathrm{d}}$ is obtained, i.e.,

$$
\begin{aligned}
P_{\mathrm{d}} \triangleq & P_{\hat{P} / H_{1}}(x)>\mu \\
= & N^{2} \sum_{i=0}^{N / 2-1} \frac{A_{i}^{2}}{\alpha_{i}^{2}} \int_{\mu}^{\infty} x e^{-N x / \alpha_{i}} \\
& +2 N \sum_{i=0, i \neq j}^{N / 2-1} \sum_{j=0}^{N / 2-1} \frac{A_{i} A_{j}}{\alpha_{i}-\alpha_{j}} \int_{\mu}^{\infty} e^{-N x / \alpha_{i}} d x \\
= & \sum_{i=0}^{N / 2-1} A_{i}^{2}\left(\frac{N \mu}{\alpha_{i}}+1\right) e^{-N \mu / \alpha_{i}} \\
& +2 \sum_{i=0, i \neq j}^{N / 2-1} \sum_{j=0}^{N / 2-1} \frac{A_{i} A_{j}}{\alpha_{i}-\alpha_{j}} \alpha_{i} e^{-N \mu / \alpha_{i}}
\end{aligned}
$$

with

$$
\begin{aligned}
& \alpha_{i}=b p+\sigma\left|D_{i}^{*} D\right|^{2} \\
& A_{i}=\left(\alpha_{i}\right)^{N / 2-1} \prod_{p=0, p \neq i} N / 2-1\left(\alpha_{i}-\alpha_{p}\right)^{-1} .
\end{aligned}
$$

This formula provides an exact expression of $P_{\mathrm{d}}$ for a moving source and the quadratic receiver. The calculation of $P_{\mathrm{d}}$ for $N$ odd is achieved by using the same method, yielding a slightly more complicated expression $\left[7,7^{\prime}\right]$.

The calculation of the $P_{\mathrm{fa}}$ is quite classical since it is not changed by the source motion. A direct application of the method of the characteristic function yields

$$
\begin{aligned}
\Theta_{0, t}(u) & \triangleq E\left[\exp \left(i u \Delta_{t}\right) \mid H_{0}\right] \\
& =(1-i u \beta)^{-1}
\end{aligned}
$$

with $\beta=b p$ and whatever $t$. Now

$$
\begin{aligned}
(1-i u \beta)^{-N} & =\int_{0}^{\infty} \frac{y^{N-1} e^{-y / \beta}}{\beta^{N}(N-1) !} \\
& =\Theta_{0}(u)
\end{aligned}
$$

so, that

$$
P_{0}(x)=\frac{x^{N-1} e^{-x / \beta}}{\beta^{N}(N-1) !}
$$

and, finally

$$
P_{\hat{p} \mid H_{0}}(x)=N P_{0}(N x)=\frac{N^{N} x^{N-1} e^{-N x / \beta}}{\beta^{N}(N-1) !} .
$$

The probability of false alarm can be directly deduced from the preceding calculations since one has

$$
\begin{aligned}
P_{\mathrm{fa}} & =\int_{\mu}^{\infty} P_{\hat{\rho} \mid H_{0}}(x) d x \\
& =e^{-N \mu / \beta} \sum_{t=0}^{N-1} \frac{1}{t !}\left(\frac{N \mu}{\beta}\right)^{\mathrm{t}} \\
\beta & =b p .
\end{aligned}
$$

It is worth comparing this expression of $P_{\mathrm{fa}_{\mathrm{a}}}$ with the expression of $P_{\mathrm{d}}$ in the case of a fixed source which stands as follows:

$$
P_{\mathrm{d}}=e^{-N \mu / \alpha} \sum_{t=0}^{N-1} \frac{1}{t !}\left(\frac{N \mu}{\alpha}\right)^{\mathrm{t}}
$$

\section{APPENDIX E}

This Appendix deals with the calculation of the likelihood functional (63) for bearings association. The proof is essentially reproduced from [40]. 
Let us consider $p\left(\mathbf{G}_{t}, m_{t} \mid \mathbf{X}\right)$, then:

$$
p\left(\mathbf{G}_{t}, m_{t} \mid \mathbf{X}\right)=p\left(\mathbf{G}_{t} \mid \mathbf{X}, m_{t}\right) p\left(m_{t} \mid \mathbf{X}\right)
$$

(using the "lemma": $p(A, B \mid C)=p(A \mid B, C) p(B \mid C)$ )

$$
\begin{aligned}
= & {\left[p\left(\mathbf{G}_{t} \mid \mathbf{X}, m_{t}, m_{t}^{d}=1\right) p\left(m_{t}^{d}=1 \mid \mathbf{X}, m_{t}\right)\right.} \\
& \left.+p\left(\mathbf{G}_{t} \mid \mathbf{X}, m_{t}, m_{t}^{d}=0\right) p\left(m_{t}^{d} \mid \mathbf{X}, m_{t}\right)\right] p\left(m_{t} \mid \mathbf{X}\right) .
\end{aligned}
$$

Now, the following equalities hold (see "lemma")

$$
\begin{array}{r}
p\left(m_{t}^{d}=\delta, m_{t} \mid \mathbf{X}\right)=p\left(m_{t}^{d}=\delta \mid \mathbf{X}, m_{t}\right) p\left(m_{t} \mid \mathbf{X}\right) \\
(\delta=0 \text { or } 1)
\end{array}
$$

so, that

$$
\begin{aligned}
p\left(m_{t}^{d}=\delta \mid \mathbf{X}, m_{t}\right) & =\frac{p\left(m_{t}^{d}=\delta, m_{t} \mid \mathbf{X}\right)}{p\left(m_{t} \mid \mathbf{X}\right)} \\
& =\frac{p\left(m_{t}^{d}=\delta, m_{t}^{f}=m_{t}-\delta \mid \mathbf{X}\right)}{p\left(m_{t} \mid \mathbf{X}\right)} \\
& =\frac{p\left(m_{t}^{d}=\delta \mid \mathbf{X}\right) p\left(m_{t}^{f}=m_{t}-\delta \mid \mathbf{X}\right)}{p\left(m_{t} \mid \mathbf{X}\right)}
\end{aligned}
$$

(independence of the true detection and false alarms)

$$
=\frac{P_{\mathrm{d}}^{\delta}\left(1-P_{\mathrm{d}}\right)^{1-\delta} \mu_{u}\left(m_{t}-\delta\right)}{p\left(m_{t} \mid \mathbf{X}\right)}
$$

( $P_{\mathrm{d}}$ denoting the probability of detection).

Furthermore, one has:

$$
p\left(\mathbf{G}_{t} \mid \mathbf{X}, m_{t}, m_{t}^{d}=1\right)=\sum_{j=1}^{m_{t}} p\left(\mathbf{g}_{j, t} \mid \mathbf{X}, m_{t}^{d}=1, H_{i, j}\right)
$$

( $H_{1, j}$ represents the event: the true detection is situated in the uncertainty cell associated with $\mathbf{g}_{j, t}$ ) since the events $H_{1, j}$ are exclusive (there is at most one true detection in $U_{t}$.

Collecting (61) and (93), it comes:

$$
\begin{aligned}
p\left(\mathbf{G}_{t} \mid \mathbf{X}, m_{t}, m_{t}^{d}=1\right) & \\
= & \frac{1}{P_{U_{t}}} \sum_{j=1}^{m_{t}}(2 \pi)^{-d / 2}(\operatorname{det} \Sigma)^{-1 / 2} \\
& \times \exp \left\{-\frac{1}{2}\left\|\mathbf{g}_{j, t}-h_{j, t}(\mathbf{X})\right\|^{2}\right\} .
\end{aligned}
$$

Furthermore, one has from $\left(A_{3}\right)$ :

$$
p\left(\mathbf{G}_{t} \mid \mathbf{X}, m_{t}, m_{t}^{d}=0\right)=U^{-m_{t}} .
$$

Finally, collecting (91)-(93) and (95) yields (63).

\section{REFERENCES}

[1] Burdic, W. S. (1991)

Underwater Acoustic System Analysis (2nd ed.).

Englewood Cliffs, NJ: Prentice Hall, 1991.

[2] Kopp, L., and Thubert, D. (1986)

Bornes de Cramer-Rao en traitement d'antenne. Première partie: formalisme.

Traitement du signal, 3, 3 (1986), 111-125.
[3] Moura, J. M. F., and Baggeroer, A. B. (1978)

Passive systems theory with narrow-band and linear constraints: Part I: spatial diversity.

IEEE Journal of Oceanic Engineering, OE-3, 1 (Jan. 1978), 5-13.

[4] Schultheiss, P. M. (1979)

Locating a passive source with array measurements, a summary of results.

In Proceedings of the IEEE International Conference on Acoustic, Speech and Signal Processing, (1979), 967-970.

[5] Bangs, W. (1971)

Array processing with generalized beamformers.

Ph.D. dissertation, Yale University, New Haven, CT, 1971.

[6] Pasupathy, S., and Alford, W. J. (1980)

Range and bearing estimation in passive sonar

IEEE Transactions on Aeraspace and Electronic Systems,

AES-16, 2 (Mar. 1980), 244-249.

[7] Le Cadre, J. P., and Zugmeyer, O. (1993)

Temporal integration for array processing.

Journal of the Acoustical Society of America, 93, 3 (Mar. 1993), 1471-1481.

[7'] Zugmeyer, O. (1992)

Méthodes d'analyse spatio-temporelle de sources en mouvement.

Thèse de doctorat de l'université de Rennes I, July 7, 1992

[8] Patzewitsch, J. T., Srinath, M. D., and Black, C. I. (1978)

Nearfield performance of passive correlation processing sonars.

Journal of the Acoustical Society of America, 64, 5 (Nov. 1978), 1412-1423.

[9] Gingras, D. F., and Hobbs, S. L. (1990)

Asymptotic statistics for a generalized frequency

wavenumber estimator.

IEEE Transactions on Acoustics, Speech and Signal

Processing, 38, 5 (May 1990), 804-813.

[10] Hobbs, S. L. (1992)

Asymptotic statistic for location estimates of acoustic signals.

Journal of the Acoustical Society of America, 91, 3 (Mar. 1992), 1538-1544.

[11] Avez, A. (1983)

Calcul differentiel.

Masson (Paris), 1983.

[12] Brillinger, D. R. (1981)

Time Series: Data Analysis and Theory.

San Francisco: Holden Day, 1981.

[13] Miller, K. S. (1974)

Complex Stochastic Processes. An Introduction to Theory and Applications.

Reading, MA: Addison Wesley, 1974.

[14] Bienvenu, G., and Kopp, L. (1982)

Bias and variance effects on the eigensystem of the cross-spectral matrix.

In Proceedings of the Institute of Acoustics, Spectral Analysis and its use in Underwater Acoustics, Imperial College, London, Apr. 29-30, 1982.

[15] Engelbrecht, R. S. (1983)

Passive source localization from spatially correlated angle of arrival data.

IEEE Transactions on Acoustics, Speech and Signal

Processing, ASSP-31 (Aug. 1983), 842-846.

[16] Hassab, J. C. (1989)

Underwater Signal and Data Processing.

Boca Raton, FL: CRC Press, 1989.

[17] Weinstein, E. (1981)

Decentralization of the Gaussian maximum likelihood estimator and its applications to passive array processing. IEEE Transactions on Acoustics Speech and Signal Processing, ASSP-29, 5 (Oct. 1981), 945-951. 
[18] Moura, J. M. F. (1979)

Passive systems theory with narrow-band and linear constraints: Part II-Temporal diversity. IEEE Journal of Oceanic Engineering, OE-4, 1 (Jan. 1979), $19-30$.

[19] Moura, J. M. F. (1979)

Passive systems theory with narrow-band and linear constraints: Part III-Spatial/temporal diversity. IEEE Journal of Oceanic Engineering, OE-4, 3 (July 1979), 113-119.

[20] Torrieri, D. J. (1984)

Statistical theory of passive location systems.

IEEE Transactions on Aerospace and Electronic Systems, AES-20, 2 (Mar. 1984).

[21] Wax, M., and Kailath, T. (1985)

Decentralized processing in sensor arrays.

IEEE Transactions on Acoustics, Speech and Signal Processing, ASSP-33 (Oct. 1985), 1123-1129.

[22] Tenney, R. R., and Sandell, N. R. (1981)

Detection with distributed sensors.

IEEE Transactions on Aerospace and Electronic Systems, AES-17, 4 (1981), 641-644.

[23] Tsitsiklis, J. N. (1993)

In H. V. Poor and J. Thomas (Eds.), Decentralized

Detection in Advances in Statistical Signal Processing, Vol.

2: Signal detection.

Greenwich, CT: JAI Press, 1993.

[24] Baldit, F., and Le Cadre, J. P. (1993)

Comparative study of centralized and decentralized localization methods.

In Proc. of the IEEE International Conference on Acoustic Speech and Signal Processing, Minneapolis, 1993, Vol. IV, 69-72.

[25] Stansfield, R. G. (1947)

Statistical theory of DF fixing.

Journal of IEEE, 14, Pt III A, 15 (1947), 762-770.

[26] Gavish, M., and Weiss, A. J. (1992)

Performance analysis of bearing-only target location algorithms.

IEEE Transactions on Aerospace and Electronic Systems, 28, 3 (July 1992), 817-828.

[27] Feintuch, P. L., and Weber, C. L. (1973)

Specification and performance of passive sonar spectral estimates.

IEEE Transactions on Aerospace and Electronic Systems, (Nov. 1973), 889-900.

[28] McDonough, R. N. (1971)

A canonical form of the likelihood detector for Gaussian random vectors.

Journal of the Acoustical Society of America, 49, 2 (Part 1), (1971), 402-406.

[29] Eastwood, L. F., Jr., and Lugannani, R. (1977)

Approximate likelihood ratio detectors for linear processes.

IEEE Transactions on Information Theory, IT-23 (July

1977), 482-489.
[30] Edelblute, D. J., Fisk, J. M., and Kinnison, G. L. (1967)

Criteria for optimum-signal-detection theory for arrays. Journal of the Acoustical Society of America, 41, 1 (1967), 199-205.

[31] Liu, S. C., and Nolte, L. W. (1980)

Performance evaluation of array processors for detecting Gaussian acoustics signals.

IEEE Transactions on Acoustics, Speech and Signal Processing, ASSP-28, 3 (June 1980), 328-333.

[32] Eddy, T. W. (1980)

Maximum likelihood detection and estimation for harmonic sets.

Journal of the Acoustical Society of America, 68, 1 (July 1980), 149-157.

[33] Schulteiss, P. M., and Weinstein, E. (1981)

Lower bounds on the localization errors of a moving source observed by a passive array.

IEEE Transactions on Acoustics, Speech and Signal Processing, ASSP-29, 3 (1981), 600-607.

[34] Weinstein, E. (1982) Optimal source localization and tracking from passive array measurements.

IEEE Transactions on Acoustics, Speech and Signal Processing, ASSP-30, 1 (Feb. 1982), 69-76.

[35] Lindgren, A. G., Irza, J., and Nardone, S. C. (1986) Trajectory estimation with uncertain and nonassociated data.

IEEE Transactions on Aerospace and Electronic Systems, AES-22, 1 (Jan. 1986), 71-78.

[36] Arnold, J. F., Bar-Shalom, Y., Estrada, R., and Mucci, R. A. (1983)

Target parameter estimation using measurements acquired with a small number of sensors.

IEEE Journal of Oceanic Engineering, OE-8 (July 1983), 163-172.

[37] Durif, J., and Kopp, L. (1987)

Trajectographie par triangulation en sonar passif

Onzieme colloque GRETSI, Actes du colloque GRETSI, Nice, France, June 1-5, 1987, 317-320.

[38] Guelle, I., and Durif, J. (1988) Inter-array data association and target motion analysis. Nato Advanced Study Institute, Kingston, Canada, 1988.

[39] Jauffret, C., and Bar-Shalom, Y. (1990)

Track formation with bearing and frequency measurements in the presence of false detection.

IEEE Transactions on Aeraspace and Electronic Systems, 26, $6(1990)$, 999-1010.

[40] Jauffret, C. (1993)

Trajectographie passive, observabilité et prise en compte des fausses alarmes.

These de doctorat, université de Toulon et du Var, février 1993, Toulon, France.

[41] Nardone, S., Lindgren, A., and Gong, K. (1984)

Fundamental properties and performance of conventional bearings-only target motion analysis.

IEEE Transactions on Automatic Control, AC-29, 9 (Sept. 1984), 775-787.

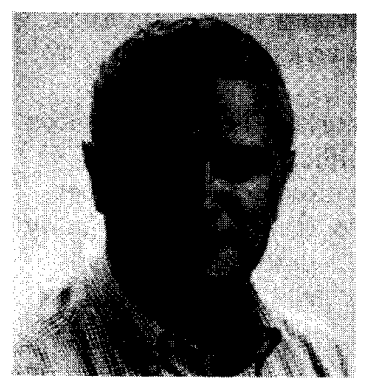

J. P. Le Cadre (M'93) received the M.S. degree in pure mathematics in 1976, the "Doctorat de 3-eme cycle" in 1982 and the "Doctorat d'Etat" in 1987, both from INPG, Grenoble, France.

From 1980 to 1989 , he worked at the GERDSM (Groupe d'Etudes et de Recherche en Detection Sous-Marines), a laboratory of the DCN (Direction des Constructions Navales), working mainly on array processing. Since 1989, he has been with IRISA/CNRS, holding a CNRS research position. His interests have now moved towards other topics like system analysis, detection, data association, and operations research. 